\title{
A speedy auction using approximated bidders' preferences
}

\author{
Jim Ingebretsen Carlson ${ }^{1}$ \\ Published online: 19 November 2019 \\ (c) The Author(s) 2019
}

\begin{abstract}
This paper presents a combinatorial auction, which is of particular interest when short completion times are of importance. It is based on a method for approximating the bidders' preferences over two types of item when complementarity between the two may exist. The resulting approximated preference relation is shown to be complete and transitive at any given price vector. It is shown that an approximated Walrasian equilibrium always exists if all bidders either view the items as substitutes or complements. If the approximated preferences of the bidders comply with the gross substitutes condition, then the set of approximated Walrasian equilibrium prices forms a complete lattice. A process is proposed that is shown to always reach the smallest approximated Walrasian price vector. Simulation results suggest that the approximation procedure works well as the difference between the approximated and true minimal Walrasian prices is small.
\end{abstract}

Keywords Approximate auction · Approximated preferences · Non-quasi-linear preferences · Combinatorial auction

\section{Introduction}

Auctions are extensively used as a way to determine who gets to buy which good and at what price. It is not uncommon for a seller to simultaneously auction multiple items. Spectrum licenses are often divided into smaller geographical areas rather than one countrywide license, and a company can be sold as several divisions rather than one entity. In recent years, the literature on multi-item auctions, and, in particular, combinatorial auctions, has grown substantially. In a unit-demand setting, Demange et al. (1986) propose a multi-item auction, which is Pareto efficient and strategy-proof. Key to their result is to find the unique minimal Walrasian equilibrium price vector, its existence being guaranteed by the lattice structure of equilibrium prices (Demange and Gale 1985; Shapley and Shubik 1972), and to allocate the items in accordance with this price. When allowing bidders to demand multiple

Financial support from the "Jan Wallander and Tom Hedelius Foundation" [P2012-0107:1] is gratefully acknowledged.

$\bowtie \quad$ Jim Ingebretsen Carlson

jim.ingebretsen_carlson@nek.uu.se

1 Department of Economics, Uppsala University, SE-751 20 Uppsala, Sweden 
units of items, the problem becomes more complex. For homogeneous items, Ausubel (2004) presents an ascending-bid auction, which is efficient and where the outcome of the auction coincides with the outcome of the Vickrey auction. Extending to heterogeneous items, Gul and Stacchetti (2000) designed a generalized version of Demange et al. (1986)'s auction, which also terminates at the unique minimal Walrasian equilibrium price vector. ${ }^{1}$ In their setting, the existence of a Walrasian equilibrium is guaranteed when bidders have gross substitute preferences. The gross substitutes condition was introduced by Kelso and Crawford (1982) and is utilized by Ausubel (2006), who suggests a multi-item auction that reaches the Vickrey-Clarkes-Groves outcome and therefore is incentive compatible. Sun and Yang (2006, 2009) introduce the gross substitutes and complements condition, which allows for some complementarity in the bidders' preferences. The authors show that this condition is sufficient for the existence of competitive equilibrium and propose two auction processes that always find an equilibrium price vector. Sun and Yang (2014) extend their work to the more general case of super additive preferences and show that an equilibrium exists when prices of the packages are allowed to be non-linear. Ausubel and Milgrom (2002) suggest an ascending-bid proxy auction: each bidder reports a valuation for each package and then commits to bid straightforwardly according to these reports. When bidders have quasi-linear preferences in money, and goods are substitutes, the outcome of the proxy auction coincides with the Vickrey auction and sincere bidding is a Nash equilibrium. By allowing prices to differ across packages and bidders, authors such as de Vries et al. (2007) and Mishra and Parkes (2007) propose auction processes that reach the VCG outcome for general valuations.

A possible problem with many auction formats is that they may take a long time to carry out. The auction for British telecom licenses, conducted in 2000, is one example of this as it took two months to complete (Binmore and Klemperer 2002). One reason for long completion times is that many auctions are dynamic processes in which the prices of the items are either only increased or only decreased. ${ }^{2}$ This may result in a time-consuming process as the starting prices have to be set far below or far above the expected final prices to make sure that the process converges to a desired equilibrium. In some cases, however, short completion times of auctions are very important. One such example is the productmix auction, which was designed to help the Bank of England during the bank run in the autumn of 2007. Due to the outbreak of the financial crisis, the Bank of England wished to allocate loans to commercial banks in a very rapid fashion. Klemperer (2010) proposed a quick auction procedure for allocating two different types of loan to the banks. The idea was that bidders submitted a number of bids consisting of two prices (interest rates), one for each type of loan, and a quantity (same for both loans), which served as an approximation of the bidders' demand. Based on the supplied quantities of the two loans, prices were determined and the bidders were awarded the loans that gave them the highest, non-negative profit. In this way, the central bank allocated the loans in a quick fashion.

Quick auctions are not uncommon in the auction literature. Sealed-bid auctions, such as the famous Vickrey auction, are well studied examples. However, such auction formats, and many more, are usually analyzed under the assumption that bidders have quasi-linear preferences in money. This may be restrictive as it implies that bidders neither exhibit risk-aversion, experience wealth effects, nor face financing- or budget constraints. If bidders' preferences are in fact non-linear in money, this should be taken into account. Optimal auctions, in which

\footnotetext{
1 Auction processes converging to the unique minimal equilibrium price vector is common in the literature; see, for example, Andersson et al. (2013), Andersson and Erlanson (2013), Mishra and Talman (2010) and Sankaran (1994).

2 For auction processes that may be both ascending and descending, see, for example, Andersson and Erlanson (2013), Ausubel (2006), Erlanson (2014) and Grigorieva et al. (2007).
} 
bidders exhibit risk-aversion, have been studied by Maskin and Riley (1984) and Matthews (1987). Morimoto and Serizawa (2015) analyzed allocation rules for multiple indivisible items, allowing bidders to have non-linear preferences in money and unit demand. Ausubel and Milgrom (2002) also propose a generalized proxy auction, in which the seller and the bidders have non-linear but strict preferences over all offers made in the bidding process. This auction is embedded in the matching with contracts model by Hatfield and Milgrom (2005).

Thus far, two problems have been identified: auctions may take a long time to conduct and bidders may not have quasi-linear preferences in money. This paper proposes a combinatorial auction which is quick and allows for bidders to have non-linear preferences in money. In order for the auction to be quick, the bidders report all required information prior to the execution of the auction. Consequently, and similar to sealed-bid auctions, the bidders do not participate in a dynamic auction process. Due to the possible high complexity of the bidders' non-linear preferences in money, requiring a bidder to report her preferences over money does not seem feasible. Therefore, the bidder will report a fraction of her preferences, which will be used to approximate her preferences. More specifically, a bidder reports two sets of prices that makes her indifferent between the packages that are available in the auction. Using these prices, linear approximations of the bidder's indifference curves between any two distinct packages will be made. In this context, an indifference curve contains all combinations of prices for the two packages, which makes the bidder indifferent between the packages. By combining the linearly approximated indifference curves, a bidder's approximated preferences can be constructed.

As suggested in the literature review, linear approximations of bidders' preferences are not uncommon. Importantly, the quasi-linear preferences are contained in the class of preferences corresponding to the approximation procedure of this paper. In particular, if a bidder has quasi-linear preferences in money and reports truthfully, the approximated preferences will coincide with the bidder's true preferences.

It is shown that the approximated preference relation of each bidder is complete and transitive at any price vector. Given the approximated preference relations of the bidders, it is of interest to know whether it is always possible to find an equilibrium assignment. In addition to theoretical interest, equilibrium assignments are particularly important in, for example, spectrum auctions as governments typically want all regions of the country to have coverage. As a bidder's approximated preferences do not necessarily coincide with her true preferences, the equilibrium concept analyzed in this paper is denoted by an approximated Walrasian equilibrium. It is shown that, if each bidder views the items as substitutes, or complements, then the set of approximated Walrasian equilibrium prices is non-empty. The substitutability (complementarity) only requires that the larger report of prices for the package of two items is strictly smaller (greater) than the sum of the larger reports for the two items separately. It is further shown that imposing the gross substitutes condition on the bidders' approximated preference relations is sufficient for the set of approximated Walrasian equilibrium prices to form a complete lattice and, hence, to contain unique minimal element. A process is described that can be used to find the unique minimal approximated Walrasian equilibrium price vector. However, the bidders do not actively participate in any intermediate step of this process. Using the bidders' approximated preferences as input, the process is a structured method for finding the unique minimal approximated Walrasian equilibrium price vector. This price vector may be of particular importance when the auctioneer is concerned with consumer welfare. A government selling spectrum licenses may be interested in assuring low consumer prices. Selling the licenses for the smallest equilibrium prices may aid in achieving this as the resulting producer costs are relatively low. Finally, simulations are conducted that 
suggest that the approximation procedure works fairly well. In fact, the absolute relative error between a true and approximated minimal Walrasian price is only $4.8 \%$ on average. This is compared to the case when bidders are assumed to have quasi-linear preferences, in which case the absolute relative error is $71.5 \%$ on average.

To summarize auction procedure can be summarized in the following steps:

1. Each bidder reports prices that makes her indifferent between the available packages.

2. These prices are used to construct linear approximations of the bidder's indifference curves.

3. Combining a bidder's linearly approximated indifference curves, her approximated preferences are constructed.

4. Using the approximated preferences as input, a process is used to find the unique minimal approximated Walrasian equilibrium price vector.

5. The items are allocated to the bidders in accordance with this price vector.

The paper is outlined as follows: Sect. 2 introduces the basic model and some definitions. The approximation procedure is described in Sect. 3. In Sect. 4, the results concerning the existence of the approximated Walrasian equilibrium are presented. Section 5 contains a description of the process and related results. The simulation results are presented in Sect. 6. Section 7 concludes the paper. All proofs are collected in the "Appendix".

\section{The model}

A finite number of bidders, collected in the set $N=\{1,2, \ldots, n\}$, participate in the auction. A seller wishes to auction two types of indivisible items, called $a$ and $b,{ }^{3}$ of which there may exist multiple copies. Let $q_{a} \geq 1$ and $q_{b} \geq 1$ denote the finite integer number of copies of each type of item. Copies of the same type are to be sold for some uniform price, $p_{a}$ or $p_{b}$ depending on the type. In order to sell the items, the seller requires at least some prices $r_{a} \geq 0$ and $r_{b} \geq 0$ for each type of item. Such prices are referred to as the seller's reservation prices and imply that $p_{a} \geq r_{a}$ and $p_{b} \geq r_{b}$. Each bidder has the outside option of not acquiring anything in the auction. The outside option is represented by a null-item, which is denoted 0 and is equal to the empty set. The price of the null-item is normalized to 0 so $p_{0}=r_{0}=0$. Each bidder is interested in acquiring, at most, one copy of item $a$ and $b$. Let $a b=\{a, b\}$ be the combination of one item of each type and let $p_{a b}=p_{a}+p_{b}$ denote its price. The sets of items that the bidders are interested in purchasing are collected in $\mathcal{I}=\{0, a, b, a b\}$ and any element $x \in \mathcal{I}$ is referred to as a package. A bidder's preferences over the packages are determined by the utility generated from consuming the packages and their prices. A consumption bundle is therefore defined to be a pair consisting of a package and a price. For any given prices of the packages, the bidders are hence interested in consuming at least one of the consumption bundles $(0,0),\left(a, p_{a}\right),\left(b, p_{b}\right)$, or $\left(a b, p_{a b}\right)$. Each bidder $i \in N$ has a preference relation, denoted $R_{i}$, over all possible consumption bundles. $R_{i}$ is complete, transitive, continuous, and finite. Let $P_{i}$ be the strict relation and $I_{i}$ the indifference relation associated with $R_{i}$. The preferences of the bidders satisfy price monotonicity; that is, for any package $x \in \mathcal{I}$ and any two prices $p_{x}^{\prime}, p_{x}^{\prime \prime} \in \mathbb{R}_{+}$, if $p_{x}^{\prime}>p_{x}^{\prime \prime}$, then $\left(x, p_{x}^{\prime \prime}\right) P_{i}\left(x, p_{x}^{\prime}\right)$. Finally, any bidder is indifferent between any two identical consumption bundles. An objective of the auction is to find an assignment of the items to the bidders such that any bidder is assigned either $0, a, b$, or $a b$.

3 To simplify the notation, we let $a$ and $b$ denote both the item and a set containing the item, i.e. $a \equiv\{a\}$ and $b \equiv\{b\}$. 
While any number of bidders can be assigned the null-item, an assignment needs to be such that the number of assigned items of any type, $a$ or $b$, does not exceed the available number of copies of the type. Formally, let $\mu: N \rightarrow \mathcal{I}$ be an assignment such that $\# N_{a} \leq q_{a}$ and $\# N_{b} \leq q_{b}$, where $N_{a}=\{i \in N \mid \mu(i) \in\{a, a b\}\}$ and $N_{b}=\{i \in N \mid \mu(i) \in\{b, a b\}\}$, and where $\mu(i)$ denotes the assignment of bidder $i \in N$.

\section{Approximation of the bidders' preferences}

In order to approximate the true preference relation, $R_{i}$, of any bidder $i \in N$, the bidder makes two reports. The first report, denoted $v$, consists of one price $v_{j} \in \mathbb{R}$ for each package $j \in\{a, b, a b\}$. Recalling that the price of the null-item is normalized to 0 , these reported prices are interpreted as the bidder being indifferent between the consumption bundles $(0,0)$, $\left(a, v_{a}\right),\left(b, v_{b}\right)$, and, $\left(a b, v_{a b}\right)$. The second report, $z$, consists of some other prices $z_{j}<v_{j}$ for each $j \in\{a, b, a b\}$. The prices in $z$ are interpreted as making the bidder indifferent between the consumption bundles $\left(a, z_{a}\right),\left(b, z_{b}\right)$, and $\left(a b, z_{a b}\right)$. Note that any price reported for $a b$ need not necessarily equal the sum of the prices reported for the individual items. Moreover, the assumptions on $R_{i}$ guarantee the existence of prices that fulfill the requirements of the reports.

Assuming that the bidders report truthfully, the two reports will be used to make linear approximations of the bidders' indifference curves between any two distinct packages. The approximations will be referred to as the bidders' approximated indifference curves. The approximated indifference curves will be constructed under the restriction that $p_{a b}=p_{a}+p_{b}$. In line with this, four constants, which are based on the two reports, are defined: $\alpha_{v}=v_{a b}-v_{b}$, $\alpha_{z}=z_{a b}-z_{b}, \beta_{v}=v_{a b}-v_{a}$, and $\beta_{z}=z_{a b}-z_{a}$. A constant $\alpha_{j}$, where $j \in\{v, z\}$, is interpreted as a price for item $a$, which would make the bidder indifferent between the consumption bundles $\left(a b, \alpha_{j}+j_{b}\right)$ and $\left(b, j_{b}\right)$, where $j_{b}$ is either the report $v_{b}$, or $z_{b}$, defined earlier. $\beta_{j}$ has the corresponding interpretation for a price of item $b$. In this way, six pairs of prices, $\left(p_{a}, p_{b}\right)$, are extracted, with the help of which the approximated indifference curves between any two packages, except 0 , are constructed.

In the following, a number of formal concepts will be introduced. In order to ease the understanding of the approximation procedure, an example will accompany these concepts. The example is depicted in Figs. 1 and 2 and is based on a bidder $i$ making the reports of $v$ and $z$ presented in Table 1 .

From the reported prices, it follows that $\alpha_{v}=6, \beta_{v}=4, \alpha_{z}=5$, and $\beta_{z}=4$. Assuming truthful reports, two pairs of prices $(10,8)$ and $(6,5)$ are obtained such that $\left(a, p_{a}\right) I_{i}\left(b, p_{b}\right)$ for bidder $i$. In addition, $(10,4)$ and $(6,4)$ are prices for which $\left(a, p_{a}\right) I_{i}\left(a b, p_{a}+p_{b}\right)$ and for $(6,8)$ and $(5,5)$ it follows that $\left(b, p_{b}\right) I_{i}\left(a b, p_{a}+p_{b}\right)$. These six pairs of prices are shown in diagram (a) of Fig. 1 and will be the basis for the linear approximation of the bidder's indifference curves.

In order to construct the approximated indifference curve between the packages $a$ and $b$, in general, the two pairs of prices $\left(v_{a}, v_{b}\right)$ and $\left(z_{a}, z_{b}\right)$ are used in constructing the following linear function:

$$
f_{1}\left(p_{a}\right)=z_{b}+\left(p_{a}-z_{a}\right)\left(\frac{v_{b}-z_{b}}{v_{a}-z_{a}}\right)
$$

$\left(v_{a}, v_{b}\right)=(10,8)$ and $\left(z_{a}, z_{b}\right)=(6,5)$ in our example, and $f_{1}$ is depicted in diagram (b) of Fig. 1. By combining an approximated indifference curve with price monotonicity, prices that make the bidder strictly prefer one consumption bundle over another consumption bundle 


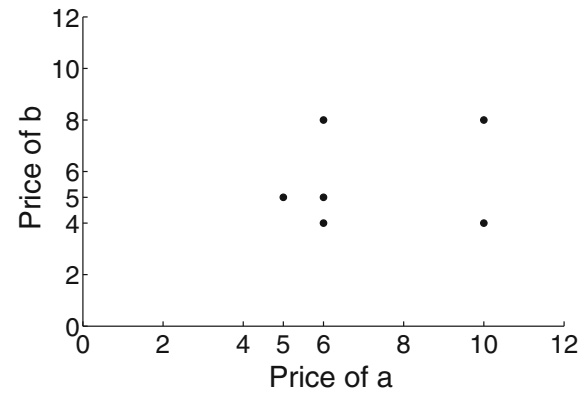

(a)

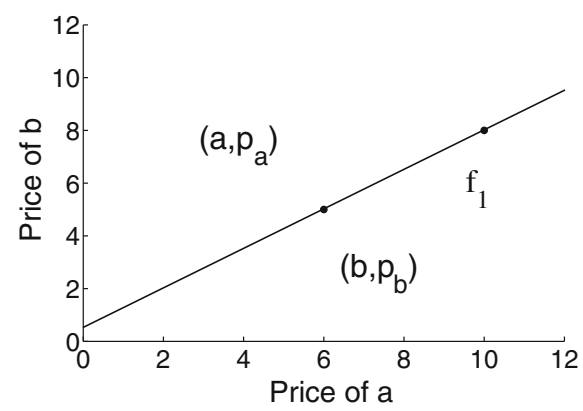

(b)

Fig. 1 First steps in approximation procedure for bidder $i$

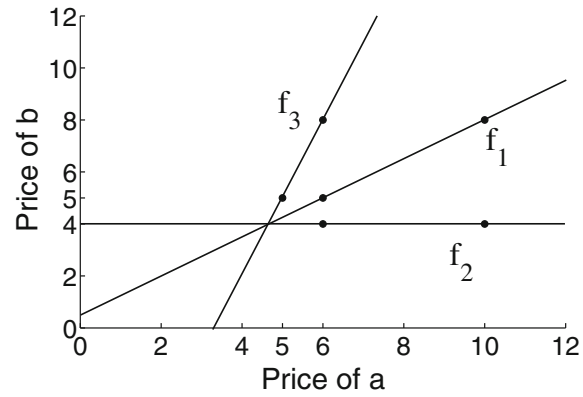

(a)

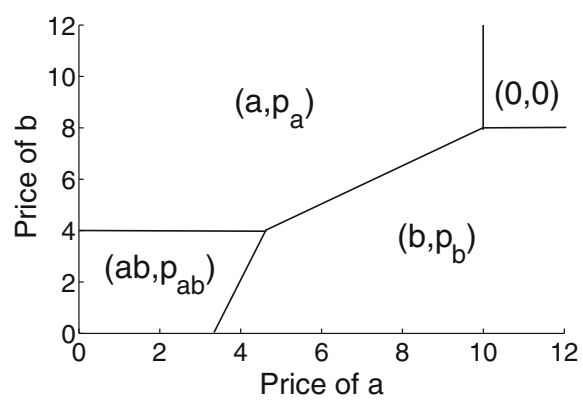

(b)

Fig. 2 Approximated indifference curves and preference relation of bidder $i$

Table 1 Reports of $v$ and $z$ by bidder $i$

\begin{tabular}{rrrrrr}
\hline & $a$ & $b$ & $a b$ & $\alpha_{j}$ & $\beta_{j}$ \\
\hline$v$ & 10 & 8 & 14 & 6 & 4 \\
$z$ & 6 & 5 & 10 & 5 & 4 \\
\hline
\end{tabular}

can be approximated. For example, as a bidder reports that she is indifferent between $\left(a, v_{a}\right)$ and $\left(b, v_{b}\right)$, it follows by price monotonicity that the bidder strictly prefers $\left(a, p_{a}\right)$ to $\left(b, p_{b}\right)$ if $p_{a} \leq v_{a}$ and $p_{b}>v_{b}$ or if $p_{a}<v_{a}$ and $p_{b} \geq v_{b}$. Similarly, prices $p_{a}$ and $p_{b}$ for which the bidder would strictly prefer $\left(b, p_{b}\right)$ to $\left(a, p_{a}\right)$ are found by reversing the inequality signs. By applying this reasoning to any pair of prices $\left(p_{a}, p_{b}\right)$ for which $f_{1}\left(p_{a}\right)=p_{b}$ is true, all pairs of prices that generate strict preferences between $\left(a, p_{a}\right)$ and $\left(b, p_{b}\right)$ are approximated. Returning to the example, diagram (b) of Fig. 1 depicts strict preferences between the consumption bundles $\left(a, p_{a}\right)$ and $\left(b, p_{b}\right) .\left(a, p_{a}\right)$ is strictly preferred to $\left(b, p_{b}\right)$ for any pair of prices above and to the left of $f_{1}$, whereas $\left(b, p_{b}\right)$ is strictly preferred to $\left(a, p_{a}\right)$ for any pair of prices below and to the right of $f_{1}$.

Similarly as for $f_{1}$, the pairs of prices $\left(v_{a}, \beta_{v}\right)$ and $\left(z_{a}, \beta_{z}\right)$ are used to construct the approximated indifference curve between the packages $a$ and $a b$, while $\left(\alpha_{v}, v_{b}\right)$ and $\left(\alpha_{z}, z_{b}\right)$ 
are used for $b$ and $a b$, in the following way:

$$
\begin{aligned}
& f_{2}\left(p_{a}\right)=\beta_{z}+\left(p_{a}-z_{a}\right)\left(\frac{\beta_{v}-\beta_{z}}{v_{a}-z_{a}}\right) \\
& f_{3}\left(p_{b}\right)=\alpha_{z}+\left(p_{b}-z_{b}\right)\left(\frac{\alpha_{v}-\alpha_{z}}{v_{b}-z_{b}}\right)
\end{aligned}
$$

The three approximated indifference curves corresponding to the bidder in our example are displayed in diagram (a) of Fig. 2. Finally, the approximated indifference curves between 0 and any other package $x$ is given by $v_{x}$. As before, by combining an approximated indifference curve and price monotonicity, strict preferences between any two consumption bundles are approximated. In this way, the approximated indifference curves and price monotonicity approximate the true preferences of a bidder. Let $\succsim_{i}$ denote the approximated preference relation of any bidder $i \in N$. Furthermore, $\succ_{i}$ and $\sim_{i}$ are the strict and indifference relations associated with $\succsim_{i}$.

In order for the approximated preference relation of a bidder to be meaningful, it is important that, at any given prices of the items, a consistent ranking of the consumption bundles can be constructed. Proposition 1 ensures that this is the case.

Proposition 1 For any given prices of the items, the approximated preference relation of each bidder $i \in N$ is complete and transitive.

Diagram (b) of Fig. 2 shows the combination of prices for which a certain consumption bundle is uniquely most preferred for the bidder in our example.

For a bidder whose preferences are quasi-linear in money, her indifference curves are linear. If prices are reported truthfully, the resulting approximated indifference curves will coincide with the true indifference curves of the bidder. The bidder's approximated and true preferences will therefore coincide and the quasi-linear preferences are thus contained in the class of preferences corresponding to the approximation procedure described in this section. It is difficult to assess how well the approximated preferences approximate the true preferences since this depends on the degree of non-linearity of the preferences in money and what prices of $z$ are reported. As long as a bidder's true indifference curves are not linear, there will exist some price $p$ and packages $x, y \in \mathcal{I}$ such that $(x, p) R_{i}(y, p)$ under the true preferences and $(x, p) \nsucc_{i}(y, p)$ under the approximated preferences. However, the further away a price vector $p$ is from a true indifference curve between packages $x$ and $y$, the more likely it is that if $(x, p) R_{i}(y, p)$, then $(x, p) \succ_{i}(y, p)$. Moreover, the results from the simulations reported in Sect. 6, perhaps, suggest that this is not a big issue.

\section{Existence}

Given the approximated preference relations of the bidders, it is interesting to know whether it is always possible to find an equilibrium assignment. A commonly analyzed equilibrium concept is the Walrasian equilibrium. However, as the approximated preferences do not necessarily coincide with the true preferences of the bidders, the equilibrium concept of this paper is denoted by an approximated Walrasian equilibrium. In order to define this formally, let a price vector be denoted by $p=\left(0, p_{a}, p_{b}\right) \in \mathbb{R}^{3}$, which contains a price for the null-item and one price for each type of item. Furthermore, the approximated demand correspondence of a bidder $i \in N$ is defined as $D_{i}(p)=\left\{x \in \mathcal{I} \mid\left(x, p_{x}\right) \succsim_{i}\left(y, p_{y}\right)\right.$ for all $\left.y \in \mathcal{I}\right\}$ at any $p$. If $x \in D_{i}(p)$, then package $x$ is said to be demanded by bidder $i \in N$. 
Definition 1 The pair $\langle p, \mu\rangle$ constitutes an approximated Walrasian equilibrium if: $(i) \mu(i) \in$ $D_{i}(p)$ for all $i \in N$ and $(i i)$ if $\# N_{x}<q_{x}$ for some $x \in a b$, then $p_{x}=r_{x}$.

Thus, a price vector $p$ and an assignment $\mu$ constitute an approximated Walrasian equilibrium if each bidder is assigned a package that she demands, and if a copy of an item remains unassigned, then the price of this type of item has to equal the seller's reservation price for the item.

An approximated Walrasian equilibrium does not always exist. For an excellent example, see Milgrom (2000) and recall that the quasi-linear preferences are a special case of the approximated preferences of this paper. However, requiring substitutability, or complementarity, in the bidders' preferences has been shown to guarantee the existence of equilibrium in the standard model. Kelso and Crawford (1982) required firms' preferences over workers to comply with the gross substitutes condition to show the existence of a core allocation. This, in turn, implies that a Walrasian equilibrium exists in Gul and Stacchetti (1999, 2000). Analyzing the simultaneous ascending auction, Milgrom (2000) showed that, if objects are mutual substitutes for the bidders, then the objects can be allocated in accordance with a competitive equilibrium. Similarly, in the matching with contracts model, a stable allocation exists if hospitals view contracts as substitutes (Hatfield and Milgrom 2005). Sun and Yang (2006, 2014) showed that an equilibrium also exists when bidders have complementary preferences. The existence of an equilibrium in the first study is guaranteed when bidders' preferences comply with the gross substitutes and complements condition and prices are linear. In the second study, the more general condition of superadditivity in bidders' preferences is shown to guarantee the existence of competitive equilibrium when non-linear pricing is used.

To ensure the existence of an approximated Walrasian equilibrium, we consider both substitutability and complementarity separately. First, we let the bidders treat the packages $a$ and $b$ as substitutes by making the assumption on the reports $v$ that $v_{a b}<v_{a}+v_{b}$ for each bidder $i \in N$. Then we look at the case of complementarity by requiring that $v_{a b}>v_{a}+v_{b}$ for each bidder $i \in N$. However, we do not have any requirements regarding the reports of $z$ in either case. Let $\mathcal{P}=\left\{p \in \mathbb{R}_{+}^{3} \mid \exists \mu\right.$ s.t. $\langle p, \mu\rangle$ is an approximated Walrasian equilibrium $\}$ be the set of approximated equilibrium prices. Proposition 2 asserts that, if $v_{a b}<v_{a}+v_{b}$ for all $i \in N$, then there exists an approximated Walrasian equilibrium.

Proposition 2 If $v_{a b}<v_{a}+v_{b}$ for each bidder $i \in N$, then the set of approximated equilibrium prices, $\mathcal{P}$, is non-empty.

Similarly, Proposition 3 states that, if $v_{a b}>v_{a}+v_{b}$ for all $i \in N$, then there exists an approximated Walrasian equilibrium.

Proposition 3 If $v_{a b}>v_{a}+v_{b}$ for each bidder $i \in N$, then the set of approximated equilibrium prices, $\mathcal{P}$, is non-empty.

It turns out that the existence of a unique minimal approximated Walrasian price vector is not guaranteed when either $v_{a b}<v_{a}+v_{b}$, or $v_{a b}>v_{a}+v_{b}$, for all $i \in N$. The reason, in the first case, is that the approximated indifference curves $f_{2}$ and $f_{3}$ may be downward-sloping for some bidder. In the second case, the indifference curve between $a b$ and 0 is downward-sloping by construction. Therefore, there may exist an infinite number of minimal approximated Walrasian price vectors along any such, downward-slooping, indifference curve. However, the gross substitutes condition ensures that neither $f_{2}$ or $f_{3}$ are downward-sloping for any bidder. Following Kelso and Crawford (1982), the gross substitutes condition is defined as: 
Definition 2 The approximated preference relation, $\succsim_{i}$, of any bidder $i \in N$, fulfills the gross substitutes condition if, for any two price vectors $p^{\prime} \geq p$ and any $x \in D_{i}(p)$, there exists $y \in D_{i}\left(p^{\prime}\right)$ such that $\left\{w \in x \mid p_{w}=p_{w}^{\prime}\right\} \subseteq y$.

The gross substitutes condition implies that a bidder's demand for an item does not decrease as the prices of any other items are raised and it guarantees that $\mathcal{P}$ forms a complete lattice. For any two price vectors $p^{\prime}, p^{\prime \prime} \in \mathbb{R}^{3}$, let the meet $p^{\prime} \wedge p^{\prime \prime}$ be defined as a vector $s \in \mathbb{R}^{3}$ with elements $s_{j}=\min \left\{p_{j}^{\prime}, p_{j}^{\prime \prime}\right\}$. Similarly, let the join $p^{\prime} \vee p^{\prime \prime}$ be a vector $h \in \mathbb{R}^{3}$ with elements $h_{j}=\max \left\{p_{j}^{\prime}, p_{j}^{\prime \prime}\right\}$. Any $S \subseteq \mathbb{R}^{3}$ forms a complete lattice if, for each $p^{\prime}, p^{\prime \prime} \in S$, $s, h \in S$.

Proposition 4 If the gross substitutes condition is fulfilled for the approximated preference relation of each bidder $i \in N$, then $\mathcal{P}$ forms a complete lattice.

Proposition 4 implies that $\mathcal{P}$ contains a unique minimal element. Let this unique minimal approximated Walrasian equilibrium price vector be denoted $p^{\text {min }}$.

\section{Process}

The proposed process can be used to find $p^{\mathrm{min}}$. It is designed as an English auction; starting at some low prices, prices are increased until $p^{\mathrm{min}}$ is reached. As mentioned in Sect. 1, the bidders do not actively participate in any intermediate step of the process. The process uses the approximated preference relations of each bidder as input in order to find $p^{\text {min }}$. As the approximated preferences are constructed prior to running the process, the process can be executed quickly.

Following Gul and Stacchetti (2000), the process will use the bidders' requirement of the different packages in order to, at least partly, determine how prices should be increased.

Definition 3 The requirement function $K_{i}: \mathcal{I} \times \mathbb{R}^{3} \rightarrow \mathbb{N}_{0}$ for each $i \in N$ is defined by:

$$
K_{i}(x, p)=\min _{y \in D_{i}(p)} \#(x \cap y) .
$$

Let $K_{N}(x, p)=\sum_{i \in N} K_{i}(x, p)$ be the bidders' aggregate requirement of any $x \in \mathcal{I}$ at some $p$. Proposition 5, below, justifies the interest in the requirement function. Most importantly, it asserts that, when, at some $p$, the bidders' aggregate requirement for each package is weakly less than the number of existing copies of the items contained in the package, it is possible to assign each bidder a package that she demands. Hence, the first condition for an approximated Walrasian equilibrium is fulfilled at $p$. As any bidder's requirement of the null-object always equals zero, let $q_{0}=0$ and naturally $q_{a b}=q_{a}+q_{b}$.

Proposition 5 For a given price vector $p$, there exists an assignment $\mu$ such that $\mu(i) \in D_{i}(p)$ for all bidders $i \in N$ if, and only if, $K_{N}(x, p) \leq q_{x}$ for all $x \in \mathcal{I}$.

Hence, if $K_{N}(x, p)>q_{x}$ for some package $x \in \mathcal{I}$, then there is more demand for the items contained in $x$, at $p$, than the number of available copies of $x$. To determine the net demand, in terms of aggregate requirement, for any package at some price vector $p$, the function $g: \mathcal{I} \times \mathbb{R}^{3} \rightarrow \mathbb{Z}: g(x, p)=K_{N}(x, p)-q_{x}$ is defined. Packages with the greatest net demand at $p$ are collected in $O(p)=\{x \in \mathcal{I} \mid g(x, p) \geq g(y, p)$ for all $y \in \mathcal{I}\}$.

Lemma $1 O(p)$ has a unique minimal element with respect to cardinality denoted $O_{*}(p)$. 
Lemma 1 is important for describing the process as whenever $O_{*}(p)$ contains any of $a, b$, or $a b$, in any step of the process, the prices of the items contained in $O_{*}(p)$ will be the main focus of the price increase.

A price increase consists of one part determining how much the prices are increased relative to each other and a second part deciding the magnitude. For the first part, $\delta(p) \in \mathbb{R}_{+}^{3}$ is introduced, which has elements $\delta_{x}(p)$ for each $x \in\{0, a, b\}$ and $p$. Let $p^{t} \in \mathbb{R}_{+}^{3}$ denote the price vector at step $t$ of the process. The magnitude of a price increase at any step $t$ is then given by $\varepsilon(t)=\sup \left\{e \mid O_{*}\left(p^{t}+e \delta\left(p^{t}\right)\right)=O_{*}\left(p^{t}\right)\right\}$. Step 1 of Process 1 checks if it is possible to assign all copies of the items. If this is not possible, it proceeds to Step 2 in which the prices of the items contained in $O_{*}(p)$ are raised by equal amounts. However, as the approximated preferences of the bidders are not necessarily quasi-linear, such a price increase may not always be possible. To solve this problem, let $x \neq y$ for $x, y \in a b$, and $l_{x}(t)=\inf \left\{\delta_{x}\left(p^{t}\right) \in \mathbb{R}_{+} \mid \delta_{0}\left(p^{t}\right)=0, \delta_{y}\left(p^{t}\right)=1\right.$, and $\left.\varepsilon(t)>0\right\}$ is defined. $l_{x}(t)$ and $\delta(p)$ are used to determine the relative price increase of the items. ${ }^{4}$

Process 1 Set $t=0$ and let $p^{0}=r$

Step 1: If $O_{*}\left(p^{t}\right)=0$ set $p^{t}=p^{T}$ and stop. Otherwise, go to Step 2.

Step 2: Let $\delta_{x}\left(p^{t}\right)=1$ if $x \in O_{*}\left(p^{t}\right)$ and 0 otherwise.

$$
I f=\left\{\begin{array}{l}
\varepsilon(t) \neq 0, \text { let } p^{t+1}=p^{t}+\varepsilon(t) \delta\left(p^{t}\right) \text { and set } t:=t+1 \text { and go to Step } 1 . \varepsilon(t)=0, \\
\text { go to Step3. }
\end{array}\right.
$$

Step 3: Let $\delta_{0}=0$ and

$$
\begin{aligned}
& \text { if }=\left\{\begin{array}{l}
a, a b \in O_{*}\left(p^{t}\right), \text { then } \delta_{a}\left(p^{t}\right)=1 \text { and } \delta_{b}\left(p^{t}\right)=l_{b}(t) . \\
b \in O_{*}\left(p^{t}\right), \text { then } \delta_{a}\left(p^{t}\right)=l_{a}(t) \text { and } \delta_{b}\left(p^{t}\right)=1 .
\end{array}\right. \\
& \text { Let } p^{t+1}=p^{t}+\varepsilon(t) \delta\left(p^{t}\right) \text { and set } t:=t+1 \text { and go to Step } 1 .
\end{aligned}
$$

Assuming that the bidders' approximated preferences fulfill the gross substitutes condition, Lemma 2 asserts that Process 1 does not get stuck at any step $t<T$.

Lemma 2 If the gross substitutes condition is fulfilled for the approximated preference relation of each bidder $i \in N$ and $\varepsilon(t)=0$ in Step 2 of Process 1, then $\varepsilon(t)>0$ in Step 3 of Process 1.

As $O_{*}\left(p^{T}\right)=0$, Proposition 5 ensures that the first condition for $p^{T}$ to yield an approximated Walrasian equilibrium is fulfilled. Assuming that each bidder's approximated preference relation complies with the gross substitutes condition, Theorem 1 states that Process 1 always converges to the unique minimal approximated Walrasian equilibrium price vector.

Theorem 1 If the gross substitutes condition is fulfilled for the approximated preference relation of each bidder $i \in N$, then Process 1 always terminates at $p^{T}=p^{\text {min }}$.

Finally, we consider an example of Process 1. One item of type $a$ and one item of type $b$ are to be sold and two bidders, $i$ and $j$, participate in the auction. By reporting $v$ and $z$,

\footnotetext{
${ }_{\varepsilon}(t), l_{x}(t)$ and $\delta(p)$ can be identified in a finite number of steps. If Process 1 proceeds to Step 3 at some $p^{\prime}$, then $f_{i}\left(p_{x}^{\prime}\right)=p_{y}^{\prime}$ for some indifference curve $i=1,2,3, x, y \in\{a b\}$ and $x \neq y$. A way to identify $l_{x}(t)$ is, thus, to order all indifference curves, for which the above holds true, from smallest to largest by their slopes, $m$. Starting with the smallest $m$, set $\delta_{0}\left(p^{\prime}\right)=0, \delta_{a}\left(p_{a}^{\prime}\right)=1$ and $\delta_{b}\left(p_{b}^{\prime}\right)=m$ and check if $\varepsilon(t)>0$. If not, continue with the second smallest $m$, and so on until $\varepsilon(t)>0$, which will happen by Lemma 2 . This gives $l_{x}(t)$ and $\delta\left(p^{\prime}\right)$. A straight line can be constructed from $\delta\left(p^{\prime}\right)$, with slope $\frac{p_{b}^{\prime}}{p_{a}^{\prime}} \cdot \varepsilon(t)$ can be identified by checking the intersection between this straight line and any indifference curve. Since there are only a finite number of indifference curves, this process terminates in a finite number of steps.
} 
Fig. 3 Price trajectory in example of Process 1

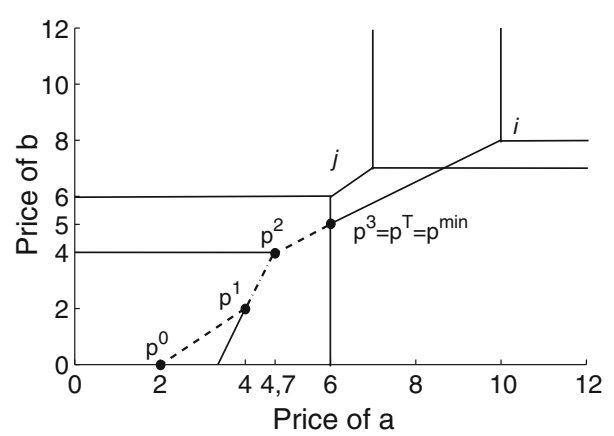

Table 2 Bidders' demand and $O_{*}\left(p^{t}\right)$ in example of Process 1

\begin{tabular}{llll}
\hline$p^{t}$ & $D_{i}\left(p^{t}\right)$ & $D_{j}\left(p^{t}\right)$ & $O_{*}\left(p^{t}\right)$ \\
\hline$p^{0}$ & $a b$ & $a b$ & $a b$ \\
$p^{1}$ & $b, a b$ & $a b$ & $b$ \\
$p^{2}$ & $a, b, a b$ & $a b$ & $a b$ \\
$p^{3}$ & $a, b$ & $b, a b$ & 0 \\
\hline
\end{tabular}

the bidders' preferences have been approximated. The parts of the bidders' approximated indifference curves that are relevant to determine their demand at any price vector are shown in Fig. 3. Note that bidder $i$ is the bidder of our example in Sect. 3. Bidder $j$ has reported $v_{a}=v_{b}=7$, and $v_{a b}=13$ as well as $z_{a}=z_{b}=5$, and $z_{a b}=11$. It is left to the reader to confirm that bidder $j$ 's reports generate the approximated indifference curves shown in Fig. 3. The seller has reservation prices $r_{a}=2$ and $r_{b}=0$ and the price trajectory of Process 1 is shown by the dashed line in Fig. 3. $O_{*}\left(p^{t}\right)$ and the packages demanded by each bidder at the price vectors corresponding to the different stages of Process 1 are shown in Table 2.

- $t=0$ : As $O_{*}\left(p^{0}\right)=\{a b\}$, Process 1 moves to Step 2 where $\delta_{a}\left(p^{0}\right)=\delta_{b}\left(p^{0}\right)=1$ and $\delta_{0}\left(p^{0}\right)=0$. Given this $\delta\left(p^{0}\right)$, it is possible to increase prices and maintain $O_{*}(p)=\{a b\}$. Consequently, $\varepsilon(0) \neq 0$ and prices are raised from $p^{0}$ to $p^{1}$ in Fig. 3. At $p^{1}, O_{*}\left(p^{1}\right)=\{b\}$ due to the change in bidder $i$ 's demand. Therefore, $p^{1}$ is the upper bound for the price increase at this step. Consequently, $p^{1}=p^{0}+\varepsilon(0) \delta\left(p^{0}\right)$ and $t=1$.

- $t=1$ : Since $O_{*}\left(p^{1}\right)=\{b\}$, we set $\delta_{b}\left(p^{1}\right)=1$ and $\delta_{0}\left(p^{1}\right)=\delta_{a}\left(p^{1}\right)=0$ in Step 2 . For this $\delta\left(p^{1}\right), \varepsilon(1)=0$ since an increase in $p_{b}$ would change $O_{*}(p)$ to contain $a b$ as $i$ would change to only demand $a b$. Therefore, Process 1 proceeds to Step 3. In this step, we find the smallest relative price increase of $p_{a}$ to $p_{b}$, which makes $\varepsilon(1) \neq 0$. In Fig. 3, this is given by the slope of the indifference curve of bidder $i . \delta_{a}\left(p^{1}\right)$ is therefore adjusted such that $\delta_{a}\left(p^{1}\right)=l_{a}(1)$, which makes $\varepsilon(1) \neq 0$. The magnitude of the price increase is bounded by the intersection of bidder $i$ 's indifference curves. This is where the demand of bidder $i$ changes. Finally, $p^{2}=p^{1}+\varepsilon(1) \delta\left(p^{1}\right)$ and $t=2$.

$-t=2$ : Now $O_{*}\left(p^{2}\right)=\{a b\}$ and the only price increase that is possible, while maintaining $O_{*}(p)=\{a b\}$, is to follow bidder $i$ 's indifference curve. $\delta\left(p^{2}\right)$ is adjusted accordingly and $p_{a}$ and $p_{b}$ are increased until the packages demanded by bidder $j$ change. Let $p^{3}=p^{2}+\varepsilon(2) \delta\left(p^{2}\right)$ and $t=3$.

- $t=3$ : $O_{*}\left(p^{3}\right)=\{0\}$ and item $a$ is sold to $i$ for a price of 6 and $b$ is sold to $j$ for a price of 5 . 


\section{Simulations}

Now that we have shown that the approximation procedure of this paper is possible to use from a practical perspective, it is natural to ask how far the unique minimal approximated Walrasian equilibrium price vector is from the true unique minimal Walrasian equilibrium price vector. Measuring this is, probably, the most relevant way to assess how well the approximation procedure of Sect. 3 approximates the bidders' true preferences since the outcome of an auction is what truly matters to bidders, sellers and auction houses. Simulations are conducted in order to measure this.

Three sets of simulations are carried out. The first set consists in calculating the true unique minimal Walrasian equilibrium price vector. Secondly, the preferences are approximated, by the procedure described in Sect. 3, to calculate the unique minimal approximated Walrasian equilibrium price vector. Thirdly, bidders are assumed to have quasi-linear preferences and are only asked to report their valuations for the packages and then the resulting unique minimal Walrasian price vector is calculated. As discussed in Sect. 4, a unique minimal approximated Walrasian price vector does not always exist since some indifference curves may be downward-sloping. This may be true for the true Walrasian price vector as well. In these cases, the minimal Walrasian equilibrium price vector that minimizes $p_{a}$ will always be picked for comparison. Calculating a true minimal Walrasian price vector is not trivial due to the non-linearity of the bidders' preferences. However, we note that such a price vector, which minimizes at least one of $p_{a}$ and $p_{b}$, must lie at the intersection of at least two indifference curves. This follows since, if this is not the case, then it would be possible to decrease the "minimal price" sufficiently little, possibly along one indifference curve, without changing any bidder's demand and, thus, still have a Walrasian equilibrium. Therefore, we calculate all prices that generate an intersection between at least two indifference curves, as well as the reservation prices, and check for the existence of an approximated Walrasian equilibrium to obtain the true (unique) minimal Walrasian price vector.

The simulations are conducted in the following setting: A seller auctions two copies of $a$ and $b$ each to four bidders. The reservation prices are set at $r_{a}=r_{b}=0$. The bidders have private valuations for the packages $0, a, b$ and $a b$, denoted by $p v_{0}^{i}, p v_{a}^{i}, p v_{b}^{i}$ and $p v_{a b}^{i}$, for any bidder $i \in N$. We let $p v_{0}^{i}=0$ for all bidders. The bidders have non-linear preferences in money and the utility for a package $x \in\{a, b, a b\}$ is given by $U_{i}(x)=p v_{x}^{i}-p_{x}^{\alpha}$, for any $i \in N$. Consequently, $\alpha$ is the parameter determining the degree of non-linearity of the bidders' preferences. We will conduct simulations for $\alpha=0.6,0.7, \ldots, 1.4$. When $\alpha>1$, bidders exhibit a special case of risk aversion known as aversion to price risk (Mezzetti 2011). Bidders are risk-neutral, and have quasi-linear preferences, when $\alpha=1$ and they are seeking price risk when $\alpha<1 .^{5} p v_{a}^{i}$ and $p v_{b}^{i}$ are randomly and independently drawn from a uniform distribution on $(10,20)$. We limit the simulations to the case when bidders view $a$ and $b$ as substitutes. Therefore, let $p v_{\max }^{i}=\max \left\{p v_{a}^{i}, p v_{b}^{i}\right\}$ for each $i \in N$. In order to ensure that $v_{a b}<v_{a}+v_{b}$, and that $a b$ is desired at some prices, $p v_{a b}^{i}$ is randomly and independently drawn from a uniform distribution on $\left(p v_{\text {max }}^{i},\left(p v_{a}^{i}+p v_{b}^{i}\right)\right.$, if $\alpha \geq 1$, and randomly and independently drawn from a uniform distribution on $\left(p v_{\text {max }}^{i},\left(p v_{a}^{i}+p v_{b}^{i}\right)^{\frac{1}{\alpha}}\right)$ otherwise. The bidders have the same private valuations for the packages in all three sets of simulations. The bidders' reports that are used for approximating their preferences are generated in the following way: Since $p_{x}=\left(p v_{x}^{i}\right)^{\frac{1}{\alpha}}$ gives $U_{i}(x)=0=U_{i}(0)$, the bidders

\footnotetext{
5 The true and approximated minimal Walrasian equilibrium prices are unique when $\alpha \leq 1$, since no indifference curves are downward-sloping. There exists no unique true or approximated minimal Walrasian equilibrium price when $\alpha>1$.
} 


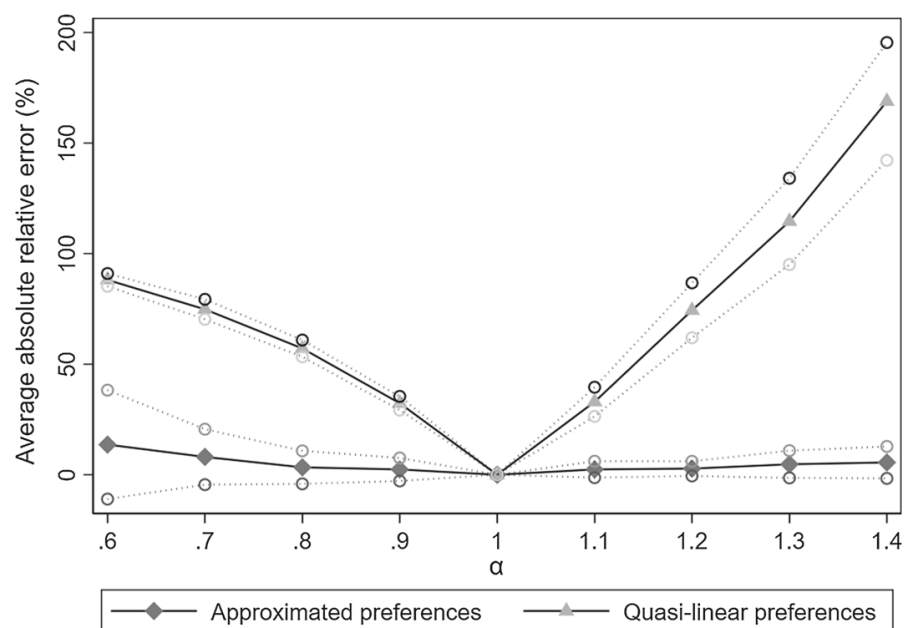

Fig. 4 Average absolute relative error (\%) for approximated (diamonds) and quasi-linear (triangles) preferences, averaged over 100 simulations, by each value of $\alpha$. Dotted lines represent standard deviations

first report $v_{x}=\left(p v_{x}^{i}\right)^{\frac{1}{\alpha}}$ for each $x \in\{a, b, a b\}$. To make sure that the reports $z$ are smaller than $v$, we let $p v_{\text {min }}^{i}=\min \left\{p v_{a}^{i}, p v_{b}^{i}\right\}$ and randomly and independently draw $c^{i}$ from a uniform distribution on $\left(0, p v_{\text {min }}^{i}\right)$ for each $i \in N$. We then let $z_{x}=\left(p v_{x}^{i}-c^{i}\right)^{\frac{1}{\alpha}}$ for each $x \in\{a, b, a b\}$ and bidder $i \in N$. The simulations were conducted using the stata 15.1 software and 100 simulations were carried out for every $\alpha$ and each of the three sets of simulations.

In order to assess performance we will, for each simulation, calculate the absolute relative error for each price $x \in\{a, b\} ; \frac{\left|p_{x}^{s}-p_{x}^{t}\right|}{p_{x}^{t}}$, and then take the average absolute relative error of the two prices. The superscripts $s$ and $t$ are used for the simulated and true prices respectively. Figure 4 shows the average absolute relative error, averaged over 100 simulations, for the approximated and quasi-linear preferences, by each value of $\alpha$.

Figure 4 suggests that the approximated Walrasian price vectors are close to the true Walrasian price vectors. In fact, the average absolute relative error is only $4.8 \%$ on average. Moreover, the quasi-linear preferences have a much larger error of $71.5 \%$ on average. The error is larger for the quasi-linear prices when $\alpha>1$, while the opposite is true for the approximated prices. As expected, there is no error for either the approximated or the quasilinear prices when $\alpha=1$. Table 3 shows the computed true, approximated and quasi-linear average equilibrium prices for each value of $\alpha$. We can conclude that the approximated equilibrium prices are close to the true equilibrium prices, while the quasi-linear prices are smaller than the true prices when $\alpha<1$ and larger when $\alpha>1$. Furthermore, the error in absolute terms is larger between the quasi-linear and true prices when $\alpha<1$.

\section{Concluding remarks}

This paper has provided a procedure for approximating a bidder's preferences over two types of items when complementarity between the two may exist. A quick auction procedure is proposed that is shown to always converge to the unique minimal approximated Walrasian 
Table 3 True, approximated and Quasi-linear average equilibrium prices by each value of $\alpha$

\begin{tabular}{|c|c|c|c|}
\hline \multirow[t]{2}{*}{$\alpha$} & \multicolumn{3}{|c|}{ Average equilibrium prices } \\
\hline & True & Approximated & Quasi-linear \\
\hline 0.6 & $\begin{array}{l}48.66 \\
(21.70)\end{array}$ & $\begin{array}{l}48.59 \\
(19.40)\end{array}$ & $\begin{array}{l}5.27 \\
(1.55)\end{array}$ \\
\hline 0.7 & $\begin{array}{l}26.75 \\
(10.59)\end{array}$ & $\begin{array}{l}27.00 \\
(9.89)\end{array}$ & $\begin{array}{l}6.39 \\
(2.08)\end{array}$ \\
\hline 0.8 & $\begin{array}{l}18.56 \\
(5.43)\end{array}$ & $\begin{array}{l}18.74 \\
(5.29)\end{array}$ & $\begin{array}{l}7.86 \\
(2.11)\end{array}$ \\
\hline 0.9 & $\begin{array}{l}13.28 \\
(3.51)\end{array}$ & $\begin{array}{l}13.38 \\
(3.46)\end{array}$ & $\begin{array}{l}8.94 \\
(2.21)\end{array}$ \\
\hline 1.0 & $\begin{array}{l}10.98 \\
(2.58)\end{array}$ & $\begin{array}{l}10.98 \\
(2.58)\end{array}$ & $\begin{array}{l}10.98 \\
(2.58)\end{array}$ \\
\hline 1.1 & $\begin{array}{l}8.10 \\
(1.82)\end{array}$ & $\begin{array}{l}7.97 \\
(1.82)\end{array}$ & $\begin{array}{l}10.58 \\
(2.55)\end{array}$ \\
\hline 1.2 & $\begin{array}{l}6.08 \\
(1.23)\end{array}$ & $\begin{array}{l}6.03 \\
(1.28)\end{array}$ & $\begin{array}{l}10.54 \\
(2.64)\end{array}$ \\
\hline 1.3 & $\begin{array}{l}4.91 \\
(1.00)\end{array}$ & $\begin{array}{l}4.84 \\
(1.11)\end{array}$ & $\begin{array}{l}10.52 \\
(2.65)\end{array}$ \\
\hline 1.4 & $\begin{array}{l}3.99 \\
(0.72)\end{array}$ & $\begin{array}{l}3.99 \\
(0.81)\end{array}$ & $\begin{array}{l}10.76 \\
(2.58)\end{array}$ \\
\hline
\end{tabular}

Standard deviations within parenthesis

equilibrium price vector. The auction procedure is efficient with respect to the approximated preferences of the bidders. Simulation results suggests that the approximation procedure works fairly well as the absolute relative error between the true and approximated minimal Walrasian equilibrium prices is only $4.8 \%$ on average. For future research, it would be desirable to find a, perhaps, similar approximation procedure that can be applied to a more general setting, in which bidders are interested in more than two items. Moreover, the auction process is designed to find the unique minimal approximated Walrasian price vector. Extending the process to the cases when such a price vector is not unique, for example, when bidders view the packages as complements, as in Sun and Yang (2009, 2014), would be another direction for future research. Furthermore, the approximation procedure described in this paper assumes that bidders report truthfully and the auction procedure is not strategy-proof. Finding a strategy-proof way of conducting a quick auction, when bidders preferences are not necessarily quasi-linear, would be of great interest and importance.

Acknowledgements Open access funding provided by Uppsala University. I want to thank Federico Echenique, Jörgen Kratz, Jens Gudmundsson, and especially Tommy Andersson for their helpful comments and suggestions.

Open Access This article is distributed under the terms of the Creative Commons Attribution 4.0 International License (http://creativecommons.org/licenses/by/4.0/), which permits unrestricted use, distribution, and reproduction in any medium, provided you give appropriate credit to the original author(s) and the source, provide a link to the Creative Commons license, and indicate if changes were made. 


\section{Appendix A: proofs related to the approximation}

For proving Proposition 1, completeness of $\succsim_{i}$ for any $i \in N$ will be shown in Lemma 3. Then Lemma 4, which is of technical nature, will be proven to aid in the proof of the transitivity of $\succsim_{i}$. Transitivity of $\succsim_{i}$ will be shown in Lemma 5 .

Let the consumption set of a bidder be $Z=\mathcal{I} \times \mathbb{R}_{+}$and any consumption bundle is a pair $\left(x, p_{x}\right) \in Z$. Let $Z(p)$ denote the consumption set at any $p=\left(p_{0}, p_{a}, p_{b}\right) \in \mathbb{R}^{3}$. For any bidder $i \in N, \succsim_{i}$ is complete if for any given $p$ and for all $\left(x, p_{x}\right),\left(y, p_{y}\right) \in Z(p)$, we have that $\left(x, p_{y}\right) \succsim_{i}\left(y, p_{y}\right)$ or $\left(y, p_{y}\right) \succsim_{i}\left(x, p_{x}\right)$ (or both). Let $\mathcal{I}_{+}=\{a, b, a b\}$.

Lemma 3 For any given prices of the items, the approximated preference relation of each bidder $i \in N$ is complete.

Proof (Proof of Lemma 3) Fix $p=\left(p_{0}, p_{a}, p_{b}\right)$. Then as any bidder is assumed to be indifferent between two identical consumption bundles, we need to show that any pair of the four distinct consumption bundles available at $p$ are related by $\succsim_{i}$. By the requirements on the bids we know that $\left(x, v_{x}\right) \sim_{i}(0,0)$ for any $x \in \mathcal{I}_{+}$. Assume that $p_{x} \leq v_{x}$. Then it follows by price monotonicity that $\left(x, p_{x}\right) \succsim\left(x, v_{x}\right) \sim_{i}(0,0)$. By construction, $f_{i}\left(p_{j}\right)=p_{k}^{i}$, for $i=1,2,3$, are some prices of $j, k \in a b$, which would make the bidder indifferent between any two packages $x \neq y$ where $x, y \in \mathcal{I}_{+}$. Assume that $p_{j}^{i} \leq p_{j}$ for $i=1,2,3$, which by price monotonicity implies that $\left(x, p_{x}\right) \succsim\left(x, p_{x}^{i}\right) \sim_{i}\left(y, p_{y}^{i}\right) \sim_{i}\left(y, p_{y}\right)$, where the identity of the two packages depend on the identity of $i$. By replacing $\leq$ with $\geq$ in the arguments above, the same conclusion is derived by symmetry.

While completeness of the approximated preference relations could be established by only considering one indifference curve at a time, transitivity depends on the construction of different indifference curves. Therefore, it is important to know the relationship of the approximated indifference curves. Let $c_{i}$ be the intercept, $m_{i}$ the slope of $f_{i}$ for $i=1,2,3$, $c_{4}=z_{b}-\frac{\alpha_{z}}{m_{3}}$, and $m_{4}=\frac{1}{m_{3}}$. We start by noting that since $v_{j}>z_{j}$ for $j \in a b$, it is always the case that $m_{1}=\frac{v_{b}-z_{b}}{v_{a}-z_{a}}>0$.

Lemma 4 The linearly approximated indifference curves have the following relationship:

(i) If $m_{j} \neq m_{k}$ for some $j, k=1,2,4$, then $m_{1} \neq m_{2} \neq m_{4}$

(ii) If $m_{1} \neq m_{2} \neq m_{4}$, then there exist unique $p_{a}^{*} \in \mathbb{R}$ and $p_{b}^{*} \in \mathbb{R}$ such that $f_{1}\left(p_{a}^{*}\right)=$ $f_{2}\left(p_{a}^{*}\right)=p_{b}^{*}$ and $f_{3}\left(p_{b}^{*}\right)=p_{a}^{*}$.

(iii) If $m_{3}>0$ and $m_{1} \neq m_{2} \neq m_{4}$, then $l>m_{1}>k$ for $l, k \in\left\{m_{2}, m_{4}\right\} \subset \mathbb{R}^{2}$ where $l \neq k$.

(iv) $m_{j}>-1$ for $j=2,3$.

(v) If $m_{2}>m_{1}$, then $m_{2}>m_{1}>m_{4}>0$.

(vi) If $m_{1}=m_{2}=m_{4}$, then $l \leq c_{1} \leq k$ for $l, k \in\left\{c_{2}, c_{4}\right\} \subset \mathbb{R}^{2}$ where $l \neq k$.

(vii) If $c_{j} \neq c_{k}$ for some $j, k=1,2,4$, then $c_{1} \neq c_{2} \neq c_{4}$

Proof (i) By symmetry it is enough to consider one case. Let $m_{1} \neq m_{4}$ and to derive a contradiction we assume that $m_{2}=m_{1} \neq m_{4}$, which is equivalent to $\frac{\beta_{v}-\beta_{z}}{v_{a}-z_{a}}=\frac{v_{b}-z_{b}}{v_{a}-z_{a}} \neq$ $\frac{v_{b}-z_{b}}{\alpha_{v}-\alpha_{z}}$. Therefore, $\beta_{v}-\beta_{z}=v_{b}-z_{b}$ and $v_{a}-z_{a} \neq \alpha_{v}-\alpha_{z}$. By the definition of the four constants $\beta_{v}, \alpha_{v}, \beta_{z}$, and $\alpha_{z}$ we know that

$$
\beta_{v}+v_{a}=\alpha_{v}+v_{b}
$$

and

$$
\beta_{z}+z_{a}=\alpha_{z}+z_{b}
$$


Using Eqs. (4) and (5) to replace $\alpha_{v}$ and $\alpha_{z}$ we get that $\beta_{v}-\beta_{z} \neq v_{b}-z_{b}$, which is a contradiction.

(ii) As any $f_{i}$ is a linear function for $i=1,2,3$ and $m_{1} \neq m_{2}$, there must exist a unique $p_{a}^{*}$ where $f_{1}=f_{2} \cdot f_{1}$ and $f_{2}$ are defined by Eqs. (1) and (2) respectively. This gives:

$$
p_{a}^{*}=\frac{z_{a}\left(v_{b}-\beta_{v}\right)+v_{a}\left(\beta_{z}-z_{b}\right)}{v_{b}-z_{b}-\beta_{v}+\beta_{z}}
$$

Naturally since $m_{1} \neq m_{2}$ we have $v_{b}-z_{b} \neq \beta_{v}-\beta_{z}$ and $v_{b}-z_{b}-\beta_{v}+\beta_{z} \neq 0$. Replacing $p_{a}$ in Eq. (1) by (6) gives:

$$
p_{b}^{*}=\frac{v_{b} \beta_{z}-z_{b} \beta_{v}}{v_{b}-z_{b}-\beta_{v}+\beta_{z}}
$$

We proceed by showing that $p_{a}^{*}$ and $p_{b}^{*}$ can be found for $f_{1}$ and $f_{3}$ as well. Replacing $p_{b}$ in (3) by (1) gives:

$$
p_{a}^{\prime}=\frac{z_{a} \alpha_{v}-\alpha_{z} v_{a}}{\alpha_{v}-\alpha_{z}-v_{a}+z_{a}}
$$

As $m_{1} \neq m_{4}$ it is ensured that $\alpha_{v}-\alpha_{z}-v_{a}+z_{a} \neq 0$. Replacing $p_{a}^{\prime}$ in Eq. (1) by (8) gives:

$$
p_{b}^{\prime}=\frac{z_{b}\left(\alpha_{v}-v_{a}\right)+v_{b}\left(z_{a}-\alpha_{z}\right)}{\alpha_{v}-\alpha_{z}-v_{a}+z_{a}}
$$

By using Eq. (4) in (8) as well as (5) in (9) we get $p_{a}^{\prime}=p_{a}^{*}$ and $p_{b}^{\prime}=p_{b}^{*}$.

(iii) First note that if $m_{3}>0$, then $m_{4}>0$. As $m_{1} \neq m_{2} \neq m_{4}$ we either have $m_{1}>m_{j}$ or $m_{1}<m_{j}$ for some $j=2,4$. By symmetry it is enough to consider one case. Let $m_{1}>m_{4}$, then $m_{1}=\frac{v_{b}-z_{b}}{v_{a}-z_{a}}>\frac{v_{b}-z_{b}}{\alpha_{v}-\alpha_{z}}=m_{4}>0$. As $v_{b}>z_{b}$ by construction we have $\alpha_{v}-\alpha_{z}>v_{a}-z_{a}$. Using Eqs. (4) and (5) to replace $\alpha_{v}$ and $\alpha_{z}$ we get $\beta_{v}-\beta_{z}>v_{b}-z_{b}$ and thus $m_{2}=\frac{\beta_{v}-\beta_{z}}{v_{a}-z_{a}}>m_{1}=\frac{v_{b}-z_{b}}{v_{a}-z_{a}}$.

(iv) As we have a requirement on the reports that $v_{a b}>z_{a b}$ we get $v_{a b}=v_{a}+\beta_{v}=$ $v_{b}+\alpha_{v}>z_{a}+\beta_{z}=z_{b}+\alpha_{z}=z_{a b}$ or $v_{a}-z_{a}>\beta_{z}-\beta_{v}$ and $v_{b}-z_{b}>\alpha_{z}-\alpha_{v}$. Therefore, $1>\frac{\beta_{z}-\beta_{v}}{v_{a}-z_{a}}$ and $1>\frac{\alpha_{z}-\alpha_{v}}{v_{b}-z_{b}}$ or equivalently, $-1<m_{2}=\frac{\beta_{v}-\beta_{z}}{v_{a}-z_{a}}$ and $-1<m_{3}=\frac{\alpha_{v}-\alpha_{z}}{v_{b}-z_{b}}$.

(v) $m_{2}>m_{1}$ gives that $\frac{\beta_{v}-\beta_{z}}{v_{a}-z_{a}}>\frac{v_{b}-z_{b}}{v_{a}-z_{a}}>0$ or $\beta_{v}-\beta_{z}>v_{b}-z_{b}$. Moreover, $m_{2}>m_{1}$ implies that $m_{2} \neq m_{1} \neq m_{4}$. Applying (4) and (5) to $\alpha$ and $\beta_{z}$ gives that $\alpha_{v}-\alpha_{z}>v_{a}-z_{a}>0$ and thus $m_{3}=\frac{\alpha_{v}-\alpha_{z}}{v_{b}-z_{b}}>0$. The rest follows from point iii of this lemma.

(vi) Let $m_{1}=m_{2}=\frac{1}{m_{3}}=m$ and then either $c_{1} \leq l$ or $c_{1} \geq l$ for $l=c_{2}, c_{4}$. By symmetry it is enough to consider when $c_{1} \geq c_{2}$, which implies $c_{1}=z_{b}-z_{a} * m \geq \beta_{z}-z_{a} * m=c_{2}$ or $z_{b} \geq \beta_{z}$. Using (5) to replace $\beta_{z}$ gives $z_{a} \geq \alpha_{z}$ and thus $c_{4}=z_{b}-\alpha_{z} * m \geq z_{b}-z_{a} * m=c_{1}$.

(vii) If $l \neq c_{1}$ for $l=c_{2}, c_{4}$, then by symmetry it is enough to consider one case: Let $c_{2} \neq c_{1}$, which implies $z_{a} \neq \alpha_{z}$. Using (5) to replace $\alpha_{z}$ gives $\beta_{z} \neq z_{b}$ and hence $c_{4} \neq c_{1}$. By point (vi) of this lemma we must have $c_{2} \neq c_{1} \neq c_{4}$. If $c_{2} \neq c_{4}$, then by point (vi) of this lemma we have $l \geq c_{1} \geq k$ with at least one weak inequality being a strict inequality and we can use the same argument as before.

For any bidder $i \in N, \succsim_{i}$ is transitive if for any given $p$ and for all $\left(x, p_{x}\right),\left(y, p_{y}\right)$, $\left(w, p_{w}\right) \in Z(p),\left(x, p_{x}\right) \succsim_{i}\left(y, p_{y}\right)$ and $\left(y, p_{y}\right) \succsim_{i}\left(w, p_{w}\right)$ imply that $\left(x, p_{x}\right) \succsim_{i}\left(w, p_{w}\right)$.

Lemma 5 For any given prices of the items, the approximated preference relation of each bidder $i \in N$ is transitive. 
Proof As $\left(x, p_{x}\right) \sim_{i}\left(x, p_{x}\right)$ at any $p$ for any $\left(x, p_{x}\right) \in Z(p)$ it is assumed that $x \neq y \neq w$. Transitivity in any other case follows by completeness. Fix some $p=\left(p_{0}, p_{a}, p_{b}\right)$. We start by considering the case when $x, y, w \in \mathcal{I}_{+}$and then proceed to where one of $x, y$, or $w$ is equal to the null-item 0. By point (i) of Lemma 4 it follows that either $m_{1}=m_{2}=m_{4}$ or $m_{1} \neq m_{2} \neq m_{4}$. These will have to be treated separately. Assume $m_{1} \neq m_{2} \neq m_{4}$ and by point (ii) of Lemma 4 there exist $p_{a}^{*}$ and $p_{b}^{*}$ such that $\left(a, p_{a}^{*}\right) \sim_{i}\left(b, p_{b}^{*}\right) \sim_{i}\left(a b, p_{a}^{*}+p_{b}^{*}\right)$. Let $x \neq y$ for $x, y \in\{b, a b\}$, then we will show the following:

If for any $i \in N\left(a, p_{a}\right) \succsim_{i}\left(x, p_{x}\right)$ and either $(i)\left(x, p_{x}\right) \succsim_{i}\left(y, p_{y}\right)$ or $(i i)\left(y, p_{y}\right) \succsim_{i}$ $\left(a, p_{a}\right)$ at some $p$, then $(i)\left(y, p_{y}\right) \nsucc_{i}\left(a, p_{a}\right)$ or $(i i)\left(x, p_{x}\right) \nsucc_{i}\left(y, p_{y}\right)$.

By symmetry, the following arguments apply when $\succsim_{i}$ and $\nsucc$ are replaced by $\preceq_{i}$ and $\nprec_{i}$ respectively. Let $f_{X}$ be the indifference curve between $a$ and $x$ and $f_{Y}$ be the indifference curve between $y$ and $a$. Note that $X, Y \in\{1,2\}$ and $X \neq Y$ as $x \neq y$. Moreover, let $f_{X}\left(p_{a}\right)=p_{b}^{X}, f_{Y}\left(p_{a}\right)=p_{b}^{Y}$ and $f_{3}\left(p_{b}\right)=p_{a}^{3}$.

Let $V \neq W$ for $V, W \in\left\{\succsim_{i}, \succ_{i}\right\}$. In order to derive a contradiction, assume that $\left(a, p_{a}\right) \succsim_{i}\left(x, p_{x}\right),\left(x, p_{x}\right) W\left(y, p_{y}\right)$, and $\left(y, p_{y}\right) V\left(a, p_{a}\right)$ for any $i \in N$ at some $p$. By price monotonicity it follows that $p_{b}^{X} \leq p_{b} \leq p_{b}^{Y}$ and, depending on the identity of the packages, either $p_{a}^{3} \geq p_{a}$ or $p_{a}^{3} \leq p_{a}$, with some weak inequality being a strict inequality.

It will now be shown that $p_{a}^{*} \neq p_{a}$. If $p_{a}=p_{a}^{*}$, then $p_{b}^{*} \neq p_{b}$ since otherwise $\left(a, p_{a}\right) \sim_{i}\left(b, p_{b}\right) \sim_{i}\left(a b, p_{a}+p_{b}\right)$, which contradicts the assumption that bidder $i \in N$ is not indifferent between the three consumption bundles. Combining $p_{b}^{X} \leq p_{b} \leq p_{b}^{Y}$ with $p_{b}^{*} \neq p_{b}$ we get that either $p_{b}^{X} \neq p_{b}^{*}$ and/or $p_{b}^{Y} \neq p_{b}^{*}$. This together with $p_{a}=p_{a}^{*}$ imply that the slopes $m_{X}=\frac{p_{b}^{X}-p_{b}^{*}}{p_{a}-p_{a}^{*}}$ and/or $m_{Y}=\frac{p_{b}^{Y}-p_{b}^{*}}{p_{a}-p_{a}^{*}}$ would be undefined. This contradicts the requirement on the bids that $v_{a}>z_{a}$. Hence, $p_{a} \neq p_{a}^{*}$.

Assume that $p_{a}>p_{a}^{*}$. Symmetric arguments, to the ones presented below, can be used when $p_{a}<p_{a}^{*}$. As $m_{1} \neq m_{2}$ by assumption, it follows that $m_{Y}=\frac{p_{b}^{Y}-p_{b}^{*}}{p_{a}-p_{a}^{*}}>m_{X}=\frac{p_{b}^{X}-p_{b}^{*}}{p_{a}-p_{a}^{*}}$.

Case $1 y=b$. Then $m_{1}>m_{2}$ and either $m_{3}=\frac{p_{a}^{3}-p_{a}^{*}}{p_{b}-p_{b}^{*}}$ or $m_{3}=\frac{p_{a}^{*}-p_{a}^{3}}{p_{b}^{*}-p_{b}}$. By price monotonicity $y=b$ requires that $p_{a}^{3} \geq p_{a}>p_{a}^{*}$, which implies that we must have $p_{b}^{*} \neq p_{b}$ as $m_{3}$ would otherwise be undefined, contradicting that $v_{b}>z_{b}$. If $p_{b}>p_{b}^{*}$, then $m_{1}=$ $\frac{p_{b}^{Y}-p_{b}^{*}}{p_{a}-p_{a}^{*}}>m_{4}=\frac{p_{b}-p_{b}^{*}}{p_{a}^{3}-p_{a}^{*}}>0$, which contradicts point (iii) of Lemma 4. If $p_{b}^{*}>p_{b}$, then we must have that $m_{1}=\frac{p_{b}^{Y}-p_{b}^{*}}{p_{a}-p_{a}^{*}}>0>\frac{p_{b}^{*}-p_{b}}{p_{a}^{*}-p_{a}^{3}}=m_{4}=\frac{p_{b}-p_{b}^{*}}{p_{a}^{3}-p_{a}^{*}} \geq m_{2}=\frac{p_{b}^{X}-p_{b}^{*}}{p_{a}-p_{a}^{*}}$. By point (iv) of Lemma $4 m_{3}>-1$ and we have $-1>m_{4} \geq m_{2}$. This is a contradiction of point (iv) of Lemma 4.

Case 2: $y=a b$. Now $p_{a}^{3} \leq p_{a}$ and $m_{2}>m_{1}=\frac{p_{b}^{X}-p_{b}^{*}}{p_{a}-p_{a}^{*}}>0$, which requires $p_{b} \geq p_{b}^{X}>$ $p_{b}^{*}$. Then it follows by point (v) of Lemma 4 that $m_{1}=\frac{p_{b}^{X}-p_{b}^{*}}{p_{a}-p_{a}^{*}}>m_{4}=\frac{p_{b}-p_{b}^{*}}{p_{a}^{3}-p_{a}^{*}}>0$. This in turn requires $p_{b}^{*}<p_{b} \leq p_{b}^{X}$ and $p_{a}^{*}<p_{a} \leq p_{a}^{3}$ with some weak inequality being a strict inequality, which is a contradiction.

Next the case when $m_{1}=m_{2}=m_{4}=m$ is considered, which implies that we can rewrite $f_{3}\left(p_{b}\right)=p_{A}^{3}=c_{3}+p_{b} * m_{3}$ as $p_{b}=-\frac{c_{3}}{m_{3}}+\frac{p_{a}^{3}}{m_{3}}$. Note that $c_{4}=-\frac{c_{3}}{m_{3}}$ and thus $p_{b}=c_{4}+p_{a}^{3} * m$. Let $x \neq y \neq w$ for $x, y, w \in \mathcal{I}_{+}$, then the following will be shown:

If $\left(x, p_{x}\right) \succsim_{i}\left(y, p_{y}\right)$ and $\left(y, p_{y}\right) \succsim_{i}\left(w, p_{w}\right)$ for any $i \in N$ at some $p$, then $\left(w, p_{w}\right) \nsucc_{i}$ $\left(x, p_{x}\right)$.

To derive a contradiction assume that $\left(x, p_{x}\right) \succsim_{i}\left(y, p_{y}\right),\left(y, p_{y}\right) \succsim_{i}\left(w, p_{w}\right)$, and $\left(w, p_{w}\right) \succ_{i}\left(x, p_{x}\right)$ for some $i \in N$ at some $p$. Note that by price monotonicity we 
either have: (i) $f_{1}\left(p_{a}\right)=p_{b}^{1} \leq p_{b} \leq p_{b}^{2}=f_{2}\left(p_{a}\right)$ and $f_{3}\left(p_{b}\right)=p_{a}^{3} \leq p_{a}$ or (ii) $f_{1}\left(p_{a}\right)=p_{b}^{1} \geq p_{b} \geq p_{b}^{2}=f_{2}\left(p_{a}\right)$ and $f_{3}\left(p_{b}\right)=p_{a}^{3} \geq p_{a}$, with at least one weak inequality being a strict inequality. By symmetry it is enough to consider one case. Assume that the three consumption bundles are related such that $f_{1}\left(p_{a}\right)=p_{b}^{1} \leq p_{b} \leq p_{b}^{2}=f_{2}\left(p_{a}\right)$ and $f_{3}\left(p_{b}\right)=p_{a}^{3} \leq p_{a}$, with at least one weak inequality being a strict inequality. From this it follows that $p_{b}^{1}=c_{1}+p_{a} * m \leq p_{b}=c_{4}+p_{a}^{3} * m \leq c_{4}+p_{a} * m$ and $p_{b}^{1}=c_{1}+p_{a} * m \leq p_{b}^{2}=c_{2}+p_{a} * m$. Thus, $c_{1} \leq c_{4}$ and $c_{1} \leq c_{2}$. However, as at least one of the three previous mentioned weak inequalities is a strict inequality we must have that $c_{j} \neq c_{k}$ for some $j \neq k$ where $j, k \in\{1,2,4\}$. Therefore, $c_{1} \neq c_{2} \neq c_{4}$ by point vii. of Lemma 4 . Hence, $c_{1}<c_{4}$ and $c_{1}<c_{2}$, which is a contradiction of point vi. of Lemma 4.

Finally, the case when $x, y, w \in \mathcal{I}$ and where one of $x, y$, or $w$ is equal to the null-item 0 is considered. By the requirements of the reports we know that $(0,0) \sim_{i}\left(a, v_{a}\right) \sim_{i}\left(b, v_{b}\right) \sim_{i}$ $\left(a b, v_{a b}\right)$ for any $i \in N$. Let $x \neq y$ for $x, y \in a b$ and $l \neq k \neq w$ for $l, k, w \in\{0, x, a b\}$, then we will show the following:

1. If $\left(x, p_{x}\right) \succsim_{i}(0,0)$ and either $(i)\left(y, p_{y}\right) \succsim_{i}\left(x, p_{x}\right)$ or $(i i)(0,0) \succsim_{i}\left(y, p_{y}\right)$ for any $i \in N$ at some $p$, then $(i)(0,0) \nsucc_{i}\left(y, p_{y}\right)$ or $(i i)\left(y, p_{y}\right) \nsucc_{i}\left(x, p_{x}\right)$.

2. If $\left(l, p_{l}\right) \succsim_{i}\left(k, p_{k}\right)$ and $\left(k, p_{k}\right) \succsim_{i}\left(w, p_{w}\right)$ for any $i \in N$ at some $p$, then $\left(w, p_{w}\right) \nsucc_{i}$ $\left(l, p_{l}\right)$

Once again, let $V \neq W$ for $V, W \in\left\{\succsim_{i}, \succ_{i}\right\}$.

1. To derive a contradiction we assume that $\left(x, p_{x}\right) \succsim_{i}(0,0),\left(y, p_{y}\right) V\left(x, p_{x}\right)$, and $(0,0) W\left(y, p_{y}\right)$. Combining we have: $\left(y, p_{y}\right) V\left(x, p_{x}\right) \succsim_{i}(0,0) \sim_{i}\left(y, v_{y}\right) W\left(y, p_{y}\right)$. By price monotonicity we have $p_{y} \leq v_{y} \leq p_{y}$, with at least one of the weak inequalities being a strict inequality.

2. Note that $p_{a b}=p_{x}+p_{y}$. Let $f_{X}$ denote the indifference curve between $x$ and $a b$ and let $m_{X}$ denote its slope. Moreover, let $f_{X}\left(p_{x}\right)=p_{y}^{X}$ for some $p_{x}$. Assume that $\left(l, p_{l}\right) \succsim_{i}\left(k, p_{k}\right)$, $\left(k, p_{k}\right) \succsim_{i}\left(w, p_{w}\right)$, and $\left(w, p_{w}\right) \succ_{i}\left(l, p_{l}\right)$ at some $p$. By price monotonicity we either have: $p_{y}^{X} \geq p_{y}, p_{x} \leq v_{x}$, and $p_{x}+p_{y} \geq v_{a b}$, or $p_{y}^{X} \leq p_{y}, p_{x} \geq v_{x}$, and $p_{x}+p_{y} \leq x_{a b}$, with at least one weak inequality being a strict inequality as $\left(w, p_{w}\right) \succ_{i}\left(l, p_{l}\right)$. By symmetry it is enough to consider one case. So assume the consumption bundles are related such that $p_{y}^{X} \geq p_{y}, p_{x} \leq v_{x}$, and $p_{x}+p_{y} \geq v_{a b}$, with at least one weak inequality being a strict inequality. By the requirements of the bids we know that $v_{a b}=v_{x}+\eta$, where $\eta$ is equal to either $\alpha_{v}$ or $\beta_{v}$ depending on the identity of $x$. Hence, $p_{x}+p_{y} \geq v_{x}+\eta$. Therefore, $p_{y}-\eta \geq v_{x}-p_{x}$ and $p_{y}^{X}-\eta \geq v_{x}-p_{x} \geq 0$. If $v_{x}=p_{x}$, then $p_{y}^{X}=\eta$ as $f_{X}\left(v_{x}\right)=\eta$ by construction. From this it follows that $p_{y}=\eta$ as $0=p_{y}^{X}-\eta \geq p_{y}-\eta \geq 0$. Therefore, $p_{y}^{X}=p_{y}$ and $p_{x}+p_{y}=v_{a b}$. Since some of the three weak inequalities above must be a strict inequality, it must be that $p_{x}<v_{x}$, which is a contradiction. Hence, $v_{x}>p_{x}$ and as $f_{X}\left(v_{x}\right)=\eta$ we must have $m_{X}=\frac{\eta-p_{x}^{Y}}{v_{x}-p_{x}}$. Since $p_{y}-\eta \geq v_{x}-p_{x}$ and $p_{y}^{X} \geq p_{y}$ by assumption, we have $m_{X} \leq-1$, which is a contradiction.

Proposition 1 For any given prices of the items, the approximated preference relation of each bidder $i \in N$ is complete and transitive.

Proof Lemmas 3 and 5 together imply Proposition 1 


\section{Appendix B: proofs related to existence}

In the following sections, it is assumed that the gross substitutes condition is fulfilled for $\succsim_{i}$ for any $i \in N$ and if $x \subset y$, then $x$ is a proper subset of $y$. An item is said to be in excess demand if there are more bidders demanding a package containing the item than the number of copies of the item. Similarly, an item is said to be in under demand if there are less bidders demanding a package containing the item than the existing number of copies of the item.

Proposition 2 If $v_{a b}<v_{a}+v_{b}$ for each bidder $i \in N$, then the set of approximated equilibrium prices, $\mathcal{P}$, is non-empty.

Proof We start by noting that it is always possible to set $p_{a}, p_{b}$, and thus $p$, sufficiently high such that it is possible to construct an assignment $\mu$ where $\mu(i) \in D_{i}(p)$ for all $x \in a b$. Let $\mathcal{C}=\left\{p \in \mathbb{R}^{3} \mid \exists \mu\right.$ s.t. $\mu(i) \in D_{i}(p)$ for all $\left.i \in N\right\}$, which we know is non-empty. Moreover, $\mathcal{P} \subset \mathcal{C}$. To derive a contradiction it is assumed that $\mathcal{P}=\emptyset$. From this it follows that for each $p \in \mathcal{C}$ there exists some assignment $\mu$ associated with $p$ such that $\# N_{x}<q_{x}$ and $p_{x}>r_{x}$ for at least some $x \in a b$ and where $\mu(i) \in D_{i}(p)$ for all $i \in N$. Let $\mu_{p}$ denote an assignment at some price vector $p$ and $\mathcal{A}(p)=\left\{\mu \mid \mu(i) \in D_{i}(p)\right.$ for all $\left.i \in N\right\}$ be the set of assignments such that each bidder is assigned a package she demands at price vector $p$. Let $r=\left(r_{0}, r_{a}, r_{b}\right)$. As $p \geq r$, it follows that $\mathcal{C}$ contains some minimal element. Denote such a minimal element by $s$. The idea of the proof is to show that if $\mathcal{P}=\emptyset$, then $s$ cannot be a minimal element of $\mathcal{C}$.

If $p_{b}=r_{b}$ for some $p \in \mathcal{C}$, then $s_{b}=r_{b}$ for some $s$ and it must be that $\# N_{a}<q_{a}$ and $s_{a}>r_{a}$ for any $\mu_{s} \in \mathcal{A}(j)$. By symmetry, the following arguments hold when $b$ and $a$ are interchanged. For this part of the proof, price monotonicity and the continuity of the approximated indifference curves will imply that $s$ cannot be a minimal element of $\mathcal{C}$. Let $p^{\prime} \leq s$ be such that $p_{b}^{\prime}=s_{b}=r_{b}$, and $r_{a} \leq p_{a}^{\prime}<s_{a}$. By price monotonicity, the demand for item $b$ has weakly decreased at $p^{\prime}$ as compared to at $s$. Moreover, as $p_{b}^{\prime}=r_{b}=s_{b}$ we know that there does not exist excess demand for item $b$ at $p^{\prime}$. Since $p^{\prime} \notin \mathcal{C}$, it is required that there exist at least some bidder $k \in N$ for whom $\mu_{p^{\prime}} \notin D_{k}\left(p^{\prime}\right)$ at any $\mu_{p^{\prime}}$. Since the demand for item $a$ has weakly increased at any $p^{\prime}$, in comparison to $s$, it must always be possible to find some $p^{\prime}$ and $\mu_{p^{\prime}}$ where either $\# N_{a}=q_{a}$, if $p_{a}^{\prime}>r_{a}$, or $\# N_{a} \leq q_{a}$, if $p_{a}^{\prime}=r_{a}$, and where $\mu_{p^{\prime}}(i) \in D_{i}\left(p^{\prime}\right)$ for all $i \in N$. Because if there exists excess demand for item $a$ at any $p^{\prime} \leq s$ and under demand at $s$, then there exist at least two bidders who did not demand any package containing $a$ at $s$ and who only demand packages containing $a$ at $p^{\prime}$. Collect these bidders in the set $F$. By price monotonicity and since the approximated indifference curves are continuous, there must exist some price vector $p^{\prime \prime}$ such that $p^{\prime}<p^{\prime \prime}<s$ for each bidder $i \in F$ where the bidder is indifferent between a package containing $a$ and another package not containing $a$. As item $a$ is in under demand at $s$, there must exist some $p^{\prime \prime}$ where it is possible to assign $\mu_{s}(j)$ to each $j \in N \backslash\{i\}$, and in particular to each $j \in F \backslash\{i\}$, and $w \supset a$ to some $i \in F$. Therefore, $\mu_{p^{\prime \prime}}(i) \in D_{i}\left(p^{\prime \prime}\right)$ for all $i \in N$ and $p^{\prime \prime} \in \mathcal{C}$, which contradicts the minimality of $s$.

Now assume that $p_{x}>r_{x}$ for all $x \in a b$ and $p \in \mathcal{C}$, which implies that there exists at least some minimal element $s \in \mathcal{C}$ such that $p^{\prime} \notin \mathcal{C}$ for any $p^{\prime} \leq s$ where $p_{x}^{\prime}<s_{x}$ for some $x \in a b$. Once again, at $s$ we know that $\# N_{x}<q_{x}$ for at least some $x \in a b$ at any $\mu_{s} \in \mathcal{A}(s)$. Assume that $\# N_{a}<q_{a}$ and $\# N_{b} \leq q_{b}$ for some $\mu_{s} \in \mathcal{A}(s)$. By symmetry, the following arguments can be used if $a$ and $b$ are interchanged. Let $p^{\prime}$ be a price vector such that $r_{a}<p_{a}^{\prime}<s_{a}$ and $p_{b}^{\prime}=s_{b}$. As $p^{\prime} \notin \mathcal{C}$ we know that $\mu_{p^{\prime}}(i) \notin D_{i}\left(p^{\prime}\right)$ for some $i \in N$ and there exists excess demand for item $a$ and/or $b$. 
Let $s$ be such that $s_{a}$ is the minimum $p_{a}$ conditional on $s \in \mathcal{C}$ and assume that item $b$ is in excess demand at $p^{\prime}$. We will let $p^{\prime \prime}$ be a price vector such that $p_{a}^{\prime \prime}<s_{a}$ and $p_{b}^{\prime \prime}>s_{b}$ and then show that $p^{\prime \prime} \in \mathcal{C}$, which contradicts the minimality of $s$. Since $s$ is minimal, it must be that some bidders are indifferent at $s$, but not at $p^{\prime}$. The change in demand of these bidders causes the excess demand of $b$ to vanish at $s$. As $p_{b}^{\prime}=s_{b}$ we cannot have that the demand of $b$ at $s$ decreases because of that $\exists i \in N$ such that $D_{i}\left(p^{\prime}\right)=\{b\}$ and $D_{i}(s)=\{a, b\}$ or $D_{i}(s)=\{b, 0\}$. Similarly, as we assume that $v_{a b}<v_{a}+v_{b}, \nexists i \in N$ such that $D_{i}\left(p^{\prime}\right)=\{a b\}$ and $D_{i}(s)=\{a b, 0\}$ since $v_{a b}=0$ implies $v_{a}>p_{a}$ and $/$ or $v_{b}>p_{b}$. Hence, the excess demand of $b$ at $p^{\prime}$, which vanishes at $s$, must stem from that $D_{i}\left(p^{\prime}\right)=\{a b\}$ and $D_{i}(s)=\{a, a b\}$ or $D_{i}(s)=\{a, b, a b\}$ for at least some bidder $i \in N$. Collect these bidders in the set $F$. Now we will show that we can assign package $a$ to any $i \in F$ at $p^{\prime \prime}$. To achieve this we let $m_{2}^{i}$ be the slope of the indifference curve between $a$ and $a b$ for bidder $i \in N$ and $m_{2}^{\max }=\max \left\{\mid m_{2}^{i} \| i \in N\right\}$ be the slope with the largest absolute value among all bidders. When choosing $p^{\prime \prime}$, we let the ratio of the absolute price change be: $\frac{\Delta p_{b}}{\left|\Delta p_{a}\right|}>m_{2}^{\max }$. This implies that the direction of the price change is mainly driven by an increase in $p_{b}$ and that we do not cross any indifference curve between the packages $a$ and $a b$ for any $i \in N$ when moving from $s$ to $p^{\prime \prime}$. Because of this and since $p_{a}^{\prime \prime}<s_{a}$ and $p_{b}^{\prime \prime}>s_{b}$ it must be that $D_{i}\left(p^{\prime \prime}\right)=\{a\}$ for all $i \in F$ and we thus let $\mu_{p^{\prime \prime}}(i)=a$ for all $i \in F$. Moreover, we make the change from $s$ to $p^{\prime \prime}$ sufficiently small such that weakly less number of bidders $i \in N$ are assigned package $a$ at $\mu_{p^{\prime \prime}}$ than at $\mu_{s}$. This is possible since we have decreased $p_{a}$ and increased $p_{b}$ and for any bidder $i \in N$ it must be that if $a \subseteq x \in D_{i}(s)$, then $a \subseteq \mu_{s}(i)$ since otherwise it would be possible to have $\# N_{a}=q_{a}$ at $s$, which is a contradiction. Finally, since $p_{a}^{\prime \prime}<s_{a}$ and $p_{b}^{\prime \prime}>s_{b}$ and we do not cross any indifference curve between the packages $a$ and $a b$ for any $i \in N$, we do not have to assign more copies of $b$ at $p^{\prime \prime}$ than we do at $s$. Consequently, $p^{\prime \prime} \in \mathcal{C}$, which contradicts that $s_{a}$ is the minimum $p_{a}$ conditional on that $s \in \mathcal{C}$

So, it must be that $a$ is the item in excess demand at $p^{\prime}$. If $\# N_{a}<q_{a}$ for all $\mu_{s} \in \mathcal{A}(s)$, then the same argument as for the case when $s_{b}=r_{b}=p_{b}^{\prime}$ can be used to generate a contradiction.

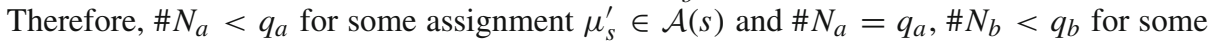
other assignment $\mu_{s}^{\prime \prime} \in \mathcal{A}(s)$ as $s \notin \mathcal{P}$. If $\# N_{b}<q_{b}$ for all $\mu_{s} \in \mathcal{A}$, then we can use symmetric arguments to case when $s_{b}=r_{b}=p_{b}^{\prime}$ in order to derive a contradiction. It must therefore be that $\# N_{a}<q_{a}$ and $\# N_{b}=q_{b}$ at $\mu_{s}^{\prime}$.

In this part it will be shown that it must be possible to find some $p^{\prime} \leq s$ such that $p^{\prime} \in \mathcal{C}$. More specifically, it will be shown that an assignment $\mu_{p^{\prime}}$ can be constructed such that $\mu_{p^{\prime}}(i) \in D_{i}\left(p^{\prime}\right)$ for all $i \in N$. To see this, note that for any bidder $i \in N$ who only demands one package, the price decrease can always be made sufficiently small such that $D_{i}\left(p^{\prime}\right)=D_{i}(s)$. For any bidder $i \in N$ for whom $0, x \in D_{i}(s)$, where $x \in\{a, b, a b\}$, then either the assumption of $v_{a b}<v_{a}+v_{b}$ is violated in the case when $x=a b$ since $v_{a b}=0$ implies $v_{a}>p_{a}$ and/or $v_{b}>p_{b}$ or it is possible to make the price decrease sufficiently small such that $x \in D_{i}\left(p^{\prime}\right)$ for any such bidder. Note that $\mu_{i}(s)=x$ at any $\mu_{s} \in \mathcal{A}(s)$ for any such bidder $i \in N$ as $s \in \mathcal{P}$ otherwise. Therefore, it is possible to construct $\mu_{p^{\prime}}$ such that $\mu_{s}(i)=\mu_{p^{\prime}}(i)=x$ for any bidder $i \in N$ discussed above. Moreover, any bidder who is indifferent between $x \in a b$ and $a b$ at $s$ must have $\mu_{s}(i)=a b$ at any $\mu_{s} \in \mathcal{A}(s)$ as $p \in \mathcal{P}$ otherwise. For any price decrease sufficiently small it follows that $D_{i}\left(p^{\prime}\right) \subseteq D_{i}\left(s^{\prime}\right)$. Hence, it is possible to let $\mu_{p^{\prime}}(i) \subseteq \mu_{s}(i)$ for any such bidder $i \in N$. The only bidders left to consider are the ones who are indifferent between $a$ and $b$. Note that some such bidder must exist as $\# N_{a}<q_{a}$ and $\# N_{b}=q_{b}$ for $\mu_{s}^{\prime}$ and $\# N_{a}=q_{a}$ and $\# N_{b}<q_{b}$ for $\mu_{s}^{\prime \prime}$. Collect each such bidder in the set $S$. As $\mu_{p^{\prime}}(i) \subseteq \mu_{s}(i)$ for all $i \in N \backslash S$ and $\# N_{x}<q_{x}$ for some $x \in a b$ at $s$, it follows that, at $p^{\prime}$, there are more copies of item $a$ and $b$ to assign to the bidders in 
$S$ than number of bidders contained in $S$. As each bidder $i \in S$ wishes to be assigned only one item at $s$ and prices can always be lowered sufficiently little such that $D_{i}\left(p^{\prime}\right) \subseteq D_{i}(s)$ for any $i \in S$, there must exist some $p^{\prime}$ where $\mu_{p^{\prime}}(i) \in D_{i}\left(p^{\prime}\right)$ for all $i \in S$.

More specifically, let $f_{1}^{i}$ be the approximated indifference curve between item $a$ and $b$ for any bidder $i \in S$ and $m_{1}^{i}$ its slope. let $T=\left\{m_{1}^{i} \mid i \in S\right\}$ and as any $m_{1}^{i} \in \mathbb{R}_{+}$, the elements in $T$ can be ordered from smallest to greatest. Let $k=\#\left\{i \in S \mid \mu_{s}^{\prime}(i)=b\right\}$. As $\# N_{a}<q_{a}$ and $\# N_{b}=q_{b}$ for $\mu_{s}^{\prime}$ and $\# N_{a}=q_{a}$ and $\# N_{b}<q_{b}$, it must be that $k \geq 1$. Pick the $k$ th element from $T$ and denote the corresponding approximated indifference curve by $f_{1}^{k}$. As $\mu_{i}\left(p^{\prime}\right)=\mu_{i}(s)$ for all $i \in N \backslash S$ it follows that $k$ is the number of copies of $b$ which are possible to assign to any bidder $i \in S$ at $p^{\prime}$. Furthermore, $\# S-k+1$ is the number of copies of $a$ which can be assigned at $p^{\prime}$. By lowering prices along $f_{1}^{k}$ sufficiently little, it must by price monotonicity be that $\left(b, p_{b}\right) \succ_{i}\left(a, p_{a}\right)$ for a maximum of $k-1$ bidders $i \in S$, $\left(a, p_{a}\right) \succ_{i}\left(b, p_{b}\right)$ for a maximum of $\# S-k$ bidders $i \in S$, and $\left(a, p_{a}\right) \sim_{i}\left(b, p_{b}\right)$ for at least 1 bidder $i \in S$. As there are more copies of item $a$ and $b$ to assign to the bidders in $S$ than number of bidders contained in $S$ at $p^{\prime}$ and no bidder requires $a b$, it is possible to let $\mu_{p^{\prime}}(i) \in D_{i}\left(p^{\prime}\right)$ for all $i \in S$. Therefore, $\mu_{p^{\prime}}(i) \in D_{i}\left(p^{\prime}\right)$ for all $i \in N$, which contradicts the minimality of $s$.

Proposition 3 If $v_{a b}>v_{a}+v_{b}$ for each bidder $i \in N$, then the set of approximated equilibrium prices, $\mathcal{P}$, is non-empty.

Proof This proof is similar to Proof 5 and will use some of the notation used in that proof. We let $s$ be a minimal element of $\mathcal{C}$. If $\mathcal{P}$ is empty, then there exists excess demand for $a$ and/or $b$ at some $q \leq s$ and under demand for $a$ and/or $b$ at $s$.

We start that noting that, since $s$ is minimal, some bidders need to be indifferent at $s$ and that these bidders cause the excess demand at $q$ to vanish at $s$. Collect these bidders in the set $F$. Note that we can make the price change sufficiently small such that $D_{i}(q)=D_{i}(s)$ for all $i \notin F$. It cannot be that $a$ and $b$ both are in excess demand since if $\{x, y\} \subseteq D_{i}(s)$ for $x \in\{a, b\}, y \in\{a b, 0\}$ and any $i \in F$, then these bidders cannot cause any under demand of $x$ at $s$. So, we must have that $\{a b, 0\} \subseteq D_{i}(s)$ for any $i \in F$, but then we can either create an approximated Walrasian equilibrium, when the number of $a$ and $b$ in excess demand are equal, or we can assign the packages such that we do not have any under demand at $s$. Since any bidder $i \in F$, previously discussed, cannot cause an under demand of $a$ or $b$ individually, at $s$, neither can them all together.

Hence, we have that either $a$ or $b$ is in excess demand $q$. Assume that $b$ is in excess demand at $q$. The same arguments can be used if $a$ is in excess demand. Select $q$ and $s$ such that $s_{a}=\min p_{a} \mid s \in \mathcal{C}$.

We will now construct $p^{\prime}$ such that $p_{a}^{\prime}<s_{a}$ and $p_{b}^{\prime}>s_{b}$ and show that $p^{\prime} \in \mathcal{C}$, contradicting the minimality of $s$. Note that it must be possible to let $\# N_{b}=q_{b}$ at $s$ since the bidders in $\mathrm{F}$, who cause the excess demand of $b$ to vanish, still demand some package containing $b$, since they are indifferent. Therefore, the same number of copies of $b$ can be assigned at $s$ as at $p$. So we can make $\# N_{b}=q_{b}$ at $s$. Consequently, $\# N_{a}<q_{a}$ for any $\mu_{s}$. Therefore, $s>r$ since otherwise $s \in \mathcal{P}$.

We make the price change from $s$ to $p^{\prime}$ sufficiently small such that any bidder only demanding one package still demands this package. We will now show that the demand for items $a$ and $b$ do not increase at $p^{\prime}$. Since \# $N_{a}<q_{a}$ for any $\mu_{s}$, it is possible to let $a \subseteq \mu_{s}$ and, thus, $a \subseteq \mu_{p^{\prime}}$ for any bidder for whom $a \subseteq D_{i}(s)$ and $a \subseteq D_{i}\left(p^{\prime}\right)$. Moreover $\nexists i \in N$ such that $a \nsubseteq D_{i}(s)$ and $a \subseteq\left(p^{\prime}\right)$. Consequently, \#N $N_{a}<q_{a}$ at $p^{\prime}$ as well.

Any bidder $i \in F$ for whom $\{b, x\} \subseteq D_{i}(s)$ for $x \in\{0, a b\}$ does not demand more copies of $b$ at $p^{\prime}$. The only buyers who can increase the demand for $b$ at $p^{\prime}$ are the ones for whom 
$\{a b, x\} \subseteq D_{i}(s)$ for $x \in\{a, 0\}$. However, we will construct $p^{\prime}$ such that these bidders can always be given $a$ or 0 . This is achieved by making the ratio of the absolute price change: $\frac{\Delta p_{b}}{\left|\Delta p_{a}\right|}$, sufficiently large such that none of the indifference curves are crossed, in which case $D_{i}\left(p^{\prime}\right)=\{a b\}$. By construction, the indifference curve between $a b$ and 0 has slope -1 . To see this, $a b \sim_{i} 0$ requires that $v_{a b}=p_{a}+p_{b}$ or $p_{b}=v_{a b}-p_{a}$, which is the expression for the indifference curve with slope $-p_{a}$. Consequently, letting $\frac{\Delta p_{b}}{\left|\Delta p_{a}\right|}>1$ ensures that any such indifference curve is not crossed. If $D_{i}(s) \subseteq\{a b, a\}$ and $D_{i}\left(p^{\prime}\right)=\{a b\}$, then the indifference curve between $a b$ and $a, f_{2}$, must be downward-sloping. Therefore, we order the indifference curves $f_{2}^{i}$ for all bidders $i \in N$ by the absolute values of their slopes $\left|m_{2}^{i}\right|$ in the set $M_{2}$. Then we let $m_{2}^{\max }=\max m_{2}^{i}|| m_{2}^{i} \mid \in M_{2}$. Finally, we let the absolute ratio of the price increase, when going from $s$ to $p^{\prime}$, be: $\frac{\Delta p_{b}}{\left|\Delta p_{a}\right|}>m_{2}^{\max }$. This ensures that we do not cross any downward sloping indifference curve $f_{2}$ and we have that $a \subseteq D_{i}\left(p^{\prime}\right)$ for any bidder $i \in F$, for whom $\{a b, a\} \subseteq D_{i}(s)$, and we can let $\mu_{i}\left(p^{\prime}\right)=a$ for any such bidder. Consequently, $\# N_{b}=q_{b}$ and $\# N_{a}<q_{a}$ at $p^{\prime}$ and $p^{\prime} \in \mathcal{C}$, which contradicts the minimality of $s_{a}$.

Lemma 6 will be used in the proof of Proposition 4.

Lemma 6 For any two price vectors $p$ and $p^{\prime}$ where $p_{x}>p_{x}^{\prime}$ and $p_{y}^{\prime} \geq p_{y}$ for $x, y \in a b$ and $x \neq y$, iffor some $i \in N, x \subseteq w$ for some $w \in D_{i}(p)$, then $x \subseteq w^{\prime}$ for all $w^{\prime} \in D_{i}\left(p^{\prime}\right)$.

Proof Let the price vector $p^{\prime \prime}$ be defined as $p_{j}^{\prime \prime}=\max \left\{p_{j}, p_{j}^{\prime}\right\}$ for all $j \in\{0, a, b\}$. Since $p_{x}^{\prime \prime}=p_{x}$ we know by gross substitutes that there exists some $w \in D_{i}\left(p^{\prime \prime}\right)$ such that $x \subseteq w$. By price monotonicity $\left(w, p_{w}^{\prime}\right) \succ_{i}\left(w, p_{w}^{\prime \prime}\right) \succsim_{i}\left(o, p_{o}^{\prime \prime}\right) \sim_{i}\left(o, p_{o}^{\prime}\right)$ for any $o \in \mathcal{I}$ for which $x \nsubseteq o$. Therefore, $x \in w^{\prime}$ for all $w^{\prime} \in D_{i}\left(p^{\prime}\right)$.

Proposition 4 If the gross substitutes condition is fulfilled for the approximated preference relation of each bidder $i \in N$, then $\mathcal{P}$ forms a complete lattice.

Proof It will first be shown that if $p^{\prime}, p^{\prime \prime} \in \mathcal{P}$, then $s \in \mathcal{P}$ and then that $h \in \mathcal{P}$ as well. Combining this with the fact that $\mathcal{P}$ is bounded from below by the seller's reservation prices and from above by some bidder's report $v$, we can conclude that $\mathcal{P}$ forms a complete lattice.

By definition $p_{0}=0$ for any $p$, so $p_{a}$ and $p_{b}$ are the prices of interest. If $\# N_{x}<q_{x}$ for some $x \in a b$ at some $p^{\prime} \in \mathcal{P}$, then we must have $p_{x}=r_{x}$ for all $p \in \mathcal{P}$. Therefore, for any $p^{\prime}, p^{\prime \prime} \in \mathcal{P}, s \in \mathcal{P}$. Now let $\left\langle p^{\prime}, \mu^{\prime}\right\rangle$ and $\left\langle p^{\prime \prime}, \mu^{\prime \prime}\right\rangle$ be two distinct approximated Walrasian equilibria where $p^{\prime}$ and $p^{\prime \prime}$ are such that $p_{a}^{\prime}>p_{a}^{\prime \prime}>r_{a}$ and $p_{b}^{\prime \prime}>p_{b}^{\prime}>r_{b}$. Hence, $\# N_{a}=q_{a}$ and $\# N_{b}=q_{b}$ for both $\mu^{\prime}$ and $\mu^{\prime \prime}$. Let $\mu_{p}$ be an assignment associated with the price vector $p$. It will first be shown that $\mu^{\prime}(i)=\mu^{\prime \prime}(i)$ for all $i \in N$ and secondly that it is possible to let $\mu^{\prime}(i)=\mu^{\prime \prime}(i)=\mu_{s}(i)=\mu_{h}(i)$ for all $i \in N$. Therefore, $\left\langle s, \mu_{s}\right\rangle$ and $\left\langle h, \mu_{h}\right\rangle$ are two approximated Walrasian equilibria.

If $\mu^{\prime}(i)=a$ for any $i \in N$, then $a \subseteq \mu^{\prime \prime}(i)$ by Lemma 6 . In order to derive a contradiction, assume $a b \in D_{i}\left(p^{\prime \prime}\right)$, which by Lemma 6 implies that $b \subseteq w$ for all $w \in D_{i}\left(p^{\prime}\right)$, which is a contradiction. Hence, $\mu^{\prime}(i)=a$ implies that $\mu^{\prime \prime}(i)=a$. Now assume $\mu^{\prime \prime}(i)=a$ and $\mu^{\prime}(i) \neq a$. Since $\# N_{a}=q_{a}$ and $\# N_{b}=q_{b}$ under both $\mu^{\prime}$ and $\mu^{\prime \prime}$, there has to exist some $j \in N \backslash\{i\}$ such that either $a \subseteq \mu^{\prime}(j)$ and $a \nsubseteq \mu^{\prime \prime}(j)$, or $b \subseteq \mu^{\prime \prime}(j)$ and $b \nsubseteq \mu^{\prime}(j)$, which we know by Lemma 6 does not exist. Therefore, $\mu^{\prime \prime}(i)=a$ implies that $\mu^{\prime}(i)=a$. If $\mu^{\prime}(i)=a b$, then $a \subseteq \mu^{\prime \prime}(i)$ by Lemma 6 , which, by using the same arguments as before, implies that $\mu^{\prime \prime}(i)=a b$. By symmetry the above arguments apply for the case when $a$ and $b$, together with the assignments, are interchanged. The previous arguments together imply that if $\mu^{\prime}(i)=0$ then $\mu^{\prime \prime}(i)=0$. 
Now to the second part. For any $i \in N$ for whom $\mu^{\prime}(i)=\mu^{\prime \prime}(i)=y$ for any $y \in\{0, a, b\}$ we know by price monotonicity that $\left(y, s_{y}\right) \succsim_{i}\left(x, s_{x}\right)$ for any $x \in\{0, a, b\}$. In order to derive a contradiction assume that $\left(a b, s_{a}+s_{b}\right) \succ_{i}\left(y, s_{y}\right)$. By gross substitutes $a \subseteq w$ for some $w \in D_{i}\left(p^{\prime \prime}\right)$ and $b \subseteq w$ for some $w \in D_{i}\left(p^{\prime}\right)$. From Lemma 6 it follows that $a b=D_{i}\left(p^{\prime \prime}\right)=$ $D_{i}\left(p^{\prime}\right)$, which is a contradiction. Finally, for any $i \in N$ for whom $\mu^{\prime}(i)=\mu^{\prime \prime}(i)=a b$, it follows by price monotonicity that $\left(a b, s_{a}+s_{b}\right) \succ_{i}(0,0),\left(a b, s_{a}+s_{b}\right) \succ_{i}\left(a b, p_{a}^{\prime}+p_{b}^{\prime}\right) \succsim_{i}$ $\left(b, p_{b}^{\prime}\right) \sim_{i}\left(b, s_{b}\right)$, and $\left(a b, s_{a}+s_{b}\right) \succ_{i}\left(a b, p_{a}^{\prime \prime}+p_{b}^{\prime \prime}\right) \succsim_{i}\left(a, p_{a}^{\prime \prime}\right) \sim_{i}\left(a, s_{a}\right)$. It is therefore possible to let $\mu(i)=a b$. Therefore, $s \in \mathcal{P}$.

Lastly it will be shown that $h \in \mathcal{P}$ as well. For any $i \in N$ for whom $\mu^{\prime}(i)=\mu^{\prime \prime}(i)=y$ for any $y \in\{0, a, b\}$ we know by price monotonicity that $\left(y, h_{y}\right) \succsim_{i}\left(x, h_{x}\right)$ for any $x \in \mathcal{I}$. If $\mu^{\prime}(i)=\mu^{\prime \prime}(i)=a b$, then $a \in w$ and $b \in w^{\prime}$ for some $w, w^{\prime} \in D_{i}(h)$ by gross substitutes. Assume $a b \notin D_{i}(h)$ and $a, b \in D_{i}(h)$. However, for any price vector $p$ such that $p_{a}<h_{a}$ and $p_{b}=h_{b}$ it follows by price monotonicity that for a price decrease sufficiently small, $b \notin D_{i}(p)$, which contradicts the gross substitutes condition. Thus, $h \in \mathcal{P}$.

\section{Appendix C: proofs related to the process}

For many of the proofs in this section, the following sets of packages are introduced: Let $C_{a}=\{a, a b,\{a, a b\}\}, C_{b}=\{b, a b,\{b, a b\}\}$ and $C_{a, b}=\{\{a, b\},\{a, b, a b\}\}$. The reason for this is that the approximated demand correspondence of any bidder who demands some package $x \neq 0$, at some $p$, is a subset of at least one of $C_{a}, C_{b}$, and $C_{a, b}$. Therefore, at any price vector $p$, it is possible to collect any bidder who demands at least some package $x \neq 0$ into at least one of the following sets: Let $\mathcal{D}_{a}(p)=\left\{i \in N \mid D_{i}(p) \in C_{a}\right\}, \mathcal{D}_{b}(p)=\{i \in N \mid$ $\left.D_{i}(p) \in C_{b}\right\}, \mathcal{D}_{a, b}(p)=\left\{i \in N \mid D_{i}(p) \in C_{a, b}\right\}$, and $\mathcal{D}_{a b}(p)=\left\{i \in N \mid D_{i}(p)=\{a b\}\right\}$. These sets will be very useful in many of the proofs in this section.

Proposition 5 For a given price vector $p$, there exists an assignment $\mu$ such that $\mu(i) \in D_{i}(p)$ for all bidders $i \in N$ if and only if $K_{N}(x, p) \leq q_{x}$ for all $x \in \mathcal{I}$.

Proof We start by showing the if part of Proposition 5: if there exists an assignment $\mu$ for some price vector $p$ such that $\mu(i) \in D_{i}(p)$ for all $i \in N$, then $K_{N}(x, p) \leq q_{x}$ for all $x \in \mathcal{I}$.

We know that $K_{N}(0, p) \leq q_{0}$ for all $p$. Note that if $K_{i}(a, p)=1$ for some $i \in N$, then $i \in \mathcal{D}_{a}(p)$. Thus, $K_{N}(a, p)=\# \mathcal{D}_{a}(p)$. Since $\mu(i) \in D_{i}(p) \forall i \in N$, it is implied that $\mathcal{D}_{a}(p) \subseteq N_{a}$. As $\# N_{a} \leq q_{a}$ by assumption, it therefore follows that $K_{N}(a, p)=\# \mathcal{D}_{a}(p) \leq$ $\# N_{a} \leq q_{a} . K_{N}(b, p) \leq q_{b}$ by symmetrical arguments.

We can also note that $K_{N}(a b, p)=\# \mathcal{D}_{a}(p)+\# \mathcal{D}_{b}(p)+\# \mathcal{D}_{a, b}(p)$ since $K_{i}(a b, p)=1$ for any $i \in N$ whenever $D_{i}(p) \in C_{a} \cup C_{b} \cup C_{a, b} \backslash a b, K_{i}(a b, p)=2$ whenever $D_{i}(p)=a b$, and $\mathcal{D}_{a}(p) \cap \mathcal{D}_{b}(p) \cap \mathcal{D}_{a, b}(p)=\mathcal{D}_{a b}(p)$. Since $\mu$ is such that $\mu(i) \in D_{i}(p)$ for all $i \in N$ by assumption, it follows that $\mathcal{D}_{a}(p) \cup \mathcal{D}_{b}(p) \cup \mathcal{D}_{a, b}(p)=N_{a} \cup N_{b}$ and $\mathcal{D}_{a b}(p) \subseteq N_{a} \cap N_{b}$. Therefore, $K_{N}(a b, p)=\# \mathcal{D}_{a}(p)+\# \mathcal{D}_{b}(p)+\# \mathcal{D}_{a, b}(p) \leq \# N_{a}+\# N_{b} \leq q_{a}+q_{b}=q_{a b}$.

We continue by showing the only if part of Proposition 5: if $K_{N}(x, p) \leq q_{x}$ for all $x \in \mathcal{I}$ at some $p$, then there exists an assignment $\mu$ such that $\mu(i) \in D_{i}(p)$ for all $i \in N$.

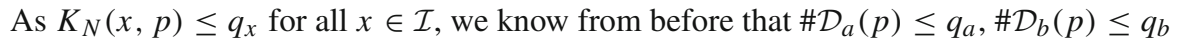
and $\# \mathcal{D}_{a}(p)+\# \mathcal{D}_{b}(p)+\# \mathcal{D}_{a, b}(p) \leq q_{a}+q_{b}$. Assume that at some price vector $p$ there does not exist a $\mu$ such that $\mu(i) \in D_{i}(p)$ for all $i \in N$, which implies that for all assignments there exists at least one bidder $i \in N$ such that $\mu(i) \notin D_{i}(p)$. Denote this bidder by $k$. Note that we can always let $\mu(k)=0$ so $k \in \mathcal{D}_{a}(p) \cup \mathcal{D}_{b}(p) \cup \mathcal{D}_{a, b}(p)$. Moreover, if $\mu(k)=a b$, then it is possible to remove items in order for $\mu(k) \in D_{k}(p)$. If there would exist a group of 
bidders $S \subseteq N$ for which $\mu(i) \notin D_{i}(p)$ for all $i \in S$, then the following arguments would apply to each bidder $i \in S$ individually.

We will focus our attention on an assignment, denoted $\mu$, for which $\# N_{x} \leq q_{x}$ for all $x \in a b$, and where each bidder $j \in N \backslash\{k\}$ is matched to a minimal element, w.r.t cardinality, of her demand correspondence. We will show, by way of contradiction, that it is always possible to construct $\mu$ such that each bidder is assigned something which she demands. As $\mu(k) \neq a b$, and $\mu(j)=a b$ if and only if $j \in \mathcal{D}_{a b}(p)$ for all $j \in N \backslash\{k\}$ we know that $\mathcal{D}_{a b}(p) \supseteq N_{a} \cap N_{b}$.

Obviously, it cannot be that $\# N_{x}<q_{x}$ for all $x \in a b$. Let $x \neq y$ for $x, y \in a b$. There are two cases to consider:

Case $1 \# N_{l}=q_{l}$ for all $l \in\{a, b\}$. We cannot have $\mu(k)=0$ because then $\mathcal{D}_{a}(p) \cup \mathcal{D}_{b}(p) \cup$ $\mathcal{D}_{a, b}(p) \supset N_{a} \cup N_{b}$ and $K_{N}(a b, p)=\# \mathcal{D}_{a}(p)+\# \mathcal{D}_{b}(p)+\# \mathcal{D}_{a, b}(p)>\# N_{a}+\# N_{b}=$ $q_{a}+q_{b}=q_{a b}$. Therefore, $\mu(k)=x$ and hence $y \subseteq w$ for all $w \in D_{k}(p)$, as we otherwise would have $\mu(k) \in D_{k}(p)$. From this it follows that $k \in \mathcal{D}_{y}$ and as $y \nsubseteq \mu(k)$ it must either be that $k \in \mathcal{D}_{a b}(p) \supset N_{a} \cap N_{b}$, in which case $K_{N}(a b, p)=\# \mathcal{D}_{a}(p)+\# \mathcal{D}_{b}(p)+\# \mathcal{D}_{a, b}(p)>$ $\# N_{a}+\# N_{b}=q_{a}+q_{b}=q_{a b}$, or $k \in \mathcal{D}_{y}(p) \backslash \mathcal{D}_{a b}(p)$, which implies that there does not exist a bidder $j \in \mathcal{D}_{a, b}(p)$ such that $y \subseteq \mu(j)$. If this was true, it would be possible to switch the assignment between bidder $k$ and bidder $j$ yielding $\mu(i) \in D_{i}(p)$ for all $i \in N$. As $y \nsubseteq \mu(j)$ for all $j \in \mathcal{D}_{a, b}(p)$, and $k \in \mathcal{D}_{y}$, it follows that $N_{y} \subset \mathcal{D}_{y}$, and thus $K_{N}(y, p)=\# \mathcal{D}_{y}(p)>\# N_{y}=q_{y}$, which is a contradiction.

Case $2 \# N_{x}<q_{x}$ and $\# N_{y}=q_{y}$. Now we can always let $\mu(i)=x$ and if $N_{x}=q_{x}$ in consequence of this, we are back in case 1 . As $\mu(k)=x \notin D_{k}(p)$ we know that $y \in w$ for all $w \in D_{k}(p)$, and $k \in \mathcal{D}_{y}$. As $\# N_{x}<q_{x}$ it is implied that there does not exist a bidder $j \in \mathcal{D}_{a, b}(p)$ such that $y \in \mu(j)$ because then it would be possible to switch the assignment between bidder $k$ and bidder $j$. Therefore, $N_{y} \subset \mathcal{D}_{y}$, and $K_{N}(y, p)=\# \mathcal{D}_{y}>\# N_{y}=q_{y}$.

Lemma $1 O(p)$ has a unique minimal element with respect to cardinality denoted $O_{*}(p)$.

Proof By the construction of $O(p)$ we know that $g(x, p)=g(y, p)$ for all $x, y \in O(p)$. Since \# $<\# a=\# b<\# a b$, we need to show that $a, b \in O_{*}(p)$ can never be true.

We will start by showing that if $x \subseteq y$ for any $x, y \in \mathcal{I}$, then $K_{i}(x) \leq K_{i}(y)$ for each $i \in N$. To derive a contradiction, assume that $x \subseteq y$ and $K_{i}(x)>K_{i}(y)$ for some $i \in N$, which is equivalent to

$$
\min _{w \in D_{i}(p)} \#(x \cap w)>\min _{w \in D_{i}(p)} \#(y \cap w)
$$

Let $w_{1} \in \arg \min _{w \in D_{i}(p)} \#(x \cap w)$ and $w_{2} \in \arg \min _{w \in D_{i}(p)} \#(y \cap w)$. If $w_{1}=w_{2}=w$, then $\#(x \cap w)>\#(y \cap w)$ implies that $x \nsubseteq \subseteq y$. If, on the other hand, $w_{1} \neq w_{2}$, then it must be that $\#\left(x \cap w_{2}\right) \geq \#\left(x \cap w_{1}\right)>\#\left(y \cap w_{2}\right)$, which in turn implies that $x \nsubseteq y$.

We will now show that $K_{i}(a b, p) \geq K_{i}(a, p)+K_{i}(b, p)$ for each $i \in N$. Since $a \subseteq a b$ and $b \subseteq a b$ it follows, by the above, that $K_{i}(a b, p) \geq \max \left\{K_{i}(a, p), K_{i}(b, p)\right\}$. Assume that $K_{i}(a b, p)<K_{i}(a, p)+K_{i}(b, p)$ for some $i \in N$ at some $p$. As $K_{i}(a, p), K_{i}(b, p) \in\{0,1\}$ we must have that $K_{i}(a, p)=K_{i}(b, p)=1$. However, $K_{i}(a, p)=K_{i}(b, p)=1$ implies that $D_{i}(p)=a b$ and thus that $K_{i}(a b, p)=K_{i}(a, p)+K_{i}(b, p)$ for each $i \in N$.

$K_{i}(a b, p) \geq K_{i}(a, p)+K_{i}(b, p)$ for each $i \in N$ implies that $K_{N}(a b, p) \geq K_{N}(a, p)+$ $K_{N}(b, p)$ as well as $g(a b, p) \geq g(a, p)+g(b, p)$. Since $g(0, p)=0$ for all $p$ we have that if $O_{*}(p)=0$, then $g(x, p) \leq 0$ for all $x \in \mathcal{I}$. So, if $a, b \in O_{*}(p)$, then $g(a, p)=$ $g(b, p)=s$ for some $s>0$ and $g(a b, p) \geq 2 s$ by the arguments above. This implies that $O(p)=O_{*}(p)=a b$, which is a contradiction.

Lemma 2 If $\varepsilon(t)=0$ in Step 2 of Process 1 , then $\varepsilon(t)>0$ in Step 3 of Process 1. 
Proof By construction of Process 1, we know that $0=O_{*}\left(p^{t}\right)$ if and only if $t=T$. So assume that $t<T, O_{*}\left(p^{t}\right)=x$ for some $x \in \mathcal{I} \backslash 0$ and that $\varepsilon\left(p^{t}\right)=0$ in step 2. It will be shown that at any $p^{t}$ there always exist some $e>0$ and $\delta\left(p^{t}\right)$ such that $O_{*}\left(p^{t}+e \delta\left(p^{t}\right)\right)=O_{*}\left(p^{t}\right)$, and hence $\varepsilon\left(p^{t}\right)>0$.

If $x=O_{*}\left(p^{t}\right) \in a b$, then by gross substitutes and price monotonicity it must be that by only raising the price of item $y$, the demand for $x$ is weakly increased and the demand for the other packages contained in $\mathcal{I} \backslash 0$ are weakly decreased. As a consequence, the aggregate requirement of $x$ weakly increases as well. Therefore, if $\delta_{0}\left(p^{t}\right)=0, \delta_{x}\left(p^{t}\right)=1$, and $\delta_{y}\left(p^{t}\right)=\infty$, then $O_{*}\left(p^{t}+e \delta\left(p^{t}\right)\right)=O_{*}\left(p^{t}\right)$ for some $e>0$ sufficiently small in step 3 of Process 1 and there exists $\varepsilon(t)>0$.

Assume $O_{*}\left(p^{t}\right)=a b$. The idea of this part of the proof is to construct a particular price vector $p^{\prime} \geq p^{t}$ and to show that the requirement for $a b=O_{*}\left(p^{t}\right)$ is greater than for any other package at $p^{\prime}$. To simplify notation, let $S=\mathcal{D}_{a, b}\left(p^{t}\right)=\left\{i \in N \mid D_{i}(p) \in C_{a, b}\right\}$. Furthermore, let $q_{x}^{S}(p)=q_{x}-K_{N \backslash S}(x, p)$ for any $x \in a b$ at some $p$. Let $p^{\prime}$ be a price vector such that $p_{x}^{\prime}>p_{x}^{t}$ for at least some $x \in a b$. Note that $K_{i}\left(a b, p^{t}\right)=K_{i}\left(a, p^{t}\right)+K_{i}\left(b, p^{t}\right)$, for any $i \in N \backslash S$ at any $p^{t}$ and that for any $p^{\prime} \geq p^{t}$ it is possible to make the price increase sufficiently small such that $K_{i}\left(a b, p^{\prime}\right)=K_{i}\left(a, p^{\prime}\right)+K_{i}\left(b, p^{\prime}\right)$ and $K_{i}\left(x, p^{\prime}\right) \geq K_{i}\left(x, p^{t}\right)$ for any $x \in \mathcal{I}$. Therefore, at any such $p^{\prime}$ it must be that $q_{a}^{S}\left(p^{\prime}\right) \leq q_{a}^{S}\left(p^{t}\right)$ and $q_{b}^{S}\left(p^{\prime}\right) \leq q_{b}^{S}\left(p^{t}\right)$. Moreover, for any $i \in S$ we have $K_{i}\left(a b, p^{t}\right)=1, K_{i}\left(x, p^{t}\right)=0$ for any $x \in \mathcal{I} \backslash a b$. Therefore, $g\left(a b, p^{t}\right)=\# S-q_{a}^{S}\left(p^{t}\right)-q_{b}^{S}\left(p^{t}\right)$.

It will now be shown that for any $p^{\prime} \geq p^{t}$, where the price increase is sufficiently small, $D_{i}\left(p^{\prime}\right) \neq\{a b\}$ for any $i \in S$. If $a b \notin D_{i}\left(p^{t}\right)$ for any $i \in S$, then any such $p^{\prime} \geq p^{t}$ can be found by making the price increase sufficiently small. If $D_{i}\left(p^{t}\right)=\{a, b, a b\}$ however, then $D_{i}\left(p^{\prime}\right)=\{a b\}$ would violate the gross substitutes condition. It can be noted that $p^{t}$ is the price vector where the three approximated indifference curves, $f_{1}, f_{2}$, and $f_{3}$, intersect for bidder $i \in S$. If $D_{i}\left(p^{\prime}\right)=a b$ for some $p^{\prime} \geq p^{t}$, then $p_{x}^{\prime}>p_{x}^{t}$ for all $x \in a b$ and we must by price monotonicity have that $f_{2}\left(p_{a}^{\prime}\right)=p_{b}^{\prime \prime}>p_{b}^{\prime}$, and $f_{3}\left(p_{b}^{\prime}\right)=p_{a}^{\prime \prime}>p_{a}^{\prime}$. Therefore, $m_{2}=\frac{p_{b}^{\prime \prime}-p_{b}^{t}}{p_{a}^{\prime}-p_{a}^{t}}>m_{4}=\frac{p_{b}^{\prime}-p_{b}^{t}}{p_{a}^{\prime \prime}-p_{a}^{t}}>0$. Let $c$ be a price vector such that $f_{2}\left(c_{a}\right)=c_{b}$ and $c_{a}+c_{b}=v_{a b}$. Since $m_{2}>m_{4}$ it must be that $f_{3}\left(c_{b}\right)=c_{a}^{\prime}>c_{a}$ and $D_{i}(c)=\{a, a b, 0\}$. Let $c^{\prime \prime}$ be a price vector such that $c_{a}^{\prime \prime}=c_{a}$ and $c_{b}^{\prime \prime}=c_{b}+\gamma$ for some $\gamma>0$. Then we must have $D_{i}\left(c^{\prime \prime}\right)=0$ for some $\gamma>0$ sufficiently small as it is always possible to find $c^{\prime \prime}$ such that $f_{3}\left(c_{b}^{\prime \prime}\right)=c_{a}^{\prime \prime \prime}>c_{a}^{\prime \prime}, c_{a}^{\prime \prime}+c_{b}^{\prime \prime}>v_{a b}$, and $f_{2}\left(c_{a}^{\prime \prime}\right)=c_{b}^{\prime \prime \prime}>c_{b}^{\prime \prime}$, which by price monotonicity implies that $(0,0) \sim_{i}\left(a b, v_{a b}\right) \succ_{i}\left(a b, c_{a}^{\prime \prime}+c_{b}^{\prime \prime}\right) \succ_{i}\left(x, c_{x}^{\prime \prime}\right)$ for $x \in a b$. However, this contradicts the gross substitutes condition as $a \nsubseteq w$ for any $w \in$ $D_{i}\left(c^{\prime \prime}\right)$.

As $D_{i}\left(p^{\prime}\right) \neq a b$ for any $i \in S$ and $p^{\prime} \geq p$, where the price increase is sufficiently small, it must be possible to construct $p^{\prime}$ such that $K_{i}\left(a b, p^{\prime}\right)=1$, for any $i \in S$. Therefore,

$$
0<g\left(a b, p^{t}\right)=\# S-q_{a}^{S}\left(p^{t}\right)-q_{b}^{S}\left(p^{t}\right) \leq \# S-q_{a}^{S}\left(p^{\prime}\right)-q_{b}^{S}\left(p^{\prime}\right)=g\left(a b, p^{\prime}\right) .
$$

The strict inequality follows from $O_{*}\left(p^{t}\right)=a b$ and the weak inequality from the fact that $q_{x}^{S}\left(p^{\prime}\right) \leq q_{x}^{S}\left(p^{t}\right)$ for $x \in a b$ and some $p^{\prime} \geq p^{t}$. So, if $q_{x}^{S}\left(p^{t}\right)<0$ for all $x \in a b$, then $g\left(a b, p^{\prime}\right)=\# S-q_{a}^{S}\left(p^{\prime}\right)-q_{b}^{S}\left(p^{\prime}\right)>\# S-q_{x}^{S}\left(p^{\prime}\right) \geq g\left(x, p^{\prime}\right)$ and $x \in a b$. The weak inequality follows from that $K_{i}\left(x, p^{\prime}\right) \in\{0,1\}$ for any $i \in S$. There are two cases two consider:

Case $1 q_{a}^{S}\left(p^{t}\right) \geq 0$ and $q_{b}^{S}\left(p^{t}\right) \geq 0$. For $g\left(a b, p^{t}\right)>0$ it has to be that $\# S>$ $q_{a}^{S}\left(p^{t}\right)+q_{b}^{S}\left(p^{t}\right)$. As before, we have $0<g\left(a b, p^{t}\right)=\# S-q_{a}^{S}\left(p^{t}\right)-q_{b}^{S}\left(p^{t}\right) \leq$ 
$g\left(a b, p^{\prime}\right)$. Let $m_{1}^{i}$ be the slope of $f_{1}^{i}$ for bidder $i \in S$, and note that $f_{1}\left(p_{a}^{t}\right)=p_{b}^{t}$ for all $i \in S$. Define $T=\left\{m_{1}^{i} \in \mathbb{R} \mid i \in S\right\}$ and let $n=q_{a}^{S}\left(p^{t}\right)+1$. Pick the $n$th element from $T$, which we denote $m_{1}^{n}$. Let $\delta_{0}\left(p^{t}\right)=0, \delta_{b}\left(p^{t}\right)=m_{1}^{n}$, and $\delta_{a}\left(p^{t}\right)=1$. By increasing the prices by $p^{\prime}=p^{t}+e \delta\left(p^{t}\right)$ for some $e>0$ sufficiently small, we must by price monotonicity have that $\left(a, p_{a}^{\prime}\right) \succ_{i}\left(b, p_{b}^{\prime}\right)$ for a maximum of $q_{a}^{S}\left(p^{t}\right)$ bidders who belong to $S,\left(b, p_{b}^{\prime}\right) \succ_{i}\left(a, p_{a}^{\prime}\right)$ for a maximum of $\# S-q_{a}^{S}\left(p^{t}\right)-1$ bidders who belong to $S$, and $\left(a, p_{a}^{\prime}\right) \sim_{i}\left(b, p_{b}^{\prime}\right)$ for at least one bidder $i \in S$. Therefore,

$$
\begin{aligned}
g\left(a, p^{\prime}\right) & \leq q_{a}^{S}\left(p^{t}\right)-q_{a}^{S}\left(p^{\prime}\right) \\
& <\# S-q_{b}^{S}\left(p^{t}\right)-q_{a}^{S}\left(p^{\prime}\right) \\
& \leq \# S-q_{b}^{S}\left(p^{\prime}\right)-q_{a}^{S}\left(p^{\prime}\right) \\
& =g\left(a b, p^{\prime}\right)
\end{aligned}
$$

The first weak inequality follows from the fact that $D_{i}\left(p^{\prime}\right) \neq a b$ for any $i \in S$. The strict inequality follows from $\# S-q_{b}^{S}\left(p^{t}\right)>q_{a}^{S}\left(p^{t}\right)$. Moreover, $g\left(b, p^{\prime}\right) \leq \# S-q_{a}^{S}\left(p^{t}\right)-1-$ $q_{b}^{S}\left(p^{\prime}\right)<\# S-q_{a}^{S}\left(p^{\prime}\right)-q_{b}^{S}\left(p^{\prime}\right)=g\left(a b, p^{\prime}\right)$. Hence, $O_{*}\left(p^{\prime}\right)=a b$, and there exist $e, \delta\left(p^{t}\right)$ such that $\varepsilon(t)>0$ in step 3 of Process 1 .

Case 2: $q_{a}^{S}\left(p^{t}\right) \geq 0$ and $q_{b}^{S}\left(p^{t}\right)<0$. For $g\left(a b, p^{t}\right)>0$ we need $g\left(a b, p^{t}\right)=\# S-$ $q_{a}^{S}\left(p^{t}\right)-q_{b}^{S}\left(p^{t}\right)>-q_{b}^{S}\left(p^{t}\right)=g\left(b, p^{t}\right)$, or $\# S>q_{a}^{S}\left(p^{t}\right)$. Moreover, $\# S>q_{a}^{S}\left(p^{t}\right) \geq q_{a}^{S}\left(p^{\prime}\right)$ from before. Let $p^{\prime}$ be such that $p_{a}^{\prime}=p_{a}^{t}$ and $p_{b}^{\prime}=p_{b}^{t}+\gamma$. Then for some $\gamma>0$ sufficiently small it must by price monotonicity be that $\left(a, p_{a}^{\prime}\right) \succ_{i}\left(b, p_{b}^{\prime}\right)$ for all $i \in S$. Combining this with $D_{i}\left(p^{\prime}\right) \neq a b$ for any $i \in S$ we have, $g\left(a, p^{\prime}\right)=\# S-q_{a}^{S}\left(p^{\prime}\right)<\# S-q_{a}^{S}\left(p^{\prime}\right)-$ $q_{b}^{S}\left(p^{\prime}\right)=g\left(a b, p^{\prime}\right)$, and $g\left(b, p^{\prime}\right)=-q_{b}^{S}\left(p^{\prime}\right)<\# S-q_{a}^{S}\left(p^{\prime}\right)-q_{b}^{S}\left(p^{\prime}\right)=g\left(a b, p^{\prime}\right)$ since $\# S>q_{a}^{S}\left(p^{\prime}\right)$. Hence, $O_{*}\left(p^{\prime}\right)=a b$, and there exist $e, \delta\left(p^{t}\right)$ such that $\varepsilon(t)>0$ in Step 3 of Process 1. Symmetric arguments can be used if $q_{b}^{S}\left(p^{t}\right) \geq 0$ and $q_{a}^{S}\left(p^{t}\right)<0$.

The proof of Theorem 1 will be decomposed into Lemmas 7 and 9. Lemma 8 will aid in the proof of Lemma 9.

Lemma $7 p^{\min } \leq p^{T}$

Proof It will be shown that for any $p \leq p^{\text {min }}$, for which $p_{x}<p_{x}^{\text {min }}$ for some $x \in a b$, it must be that $O_{*}(p) \neq 0$. As the prices are bounded from below by the seller's reservation prices it is assumed that $p_{x}^{\text {min }}>r_{x}$ for at least some $x \in a b . p$ is constructed such that $p_{x}<p_{x}^{\text {min }}$ for at least some $x \in a b$. Thus, $p \notin \mathcal{P}$.

If it is possible to construct some assignment $\mu_{p}$ at price vector $p$ such that $\# N_{x}=q_{x}$ for any $x \in a b$, or alternatively $\# N_{x}<q_{x}$ for any $x \in a b$ for which $p_{x}=r_{x}$, then there must exist $i \in N$ for whom $\mu_{p}(i) \notin D_{i}(p)$ as $p \in \mathcal{P}$ otherwise. $p \in \mathcal{P}$ would contradict the minimality of $p^{\text {min }}$. By Proposition 5 it follows that $K_{N}(x, p)>q_{x}$ for some package $x \in \mathcal{I}$ and since $K_{N}(0, p) \leq q_{0}$ for all $p$ it must be that $O_{*}(p) \neq 0$.

Now assume, in order to derive a contradiction, that $\mu_{p}$ can only be constructed such that $\# N_{x}<q_{x}$ and $p_{x}>r_{x}$ for at least some $x \in a b$ and that $\mu(i) \in D_{i}(p)$ for all $i \in N$. Then it must be possible to find a price vector $p^{\prime} \leq p$ where an assignment can be constructed such that $\mu(i) \in D_{i}\left(p^{\prime}\right)$ for all $i \in N$ and $\# N_{w}=q_{w}$ for any $w \in a b$ for which $p_{w}^{\prime}>r_{w}$ and $\# N_{w} \leq q_{w}$ for any $w \in a b$ for which $p_{w}^{\prime}=r_{w}$. To see this it can be noted that, by price monotonicity, the demand for any $w \in a b$ weakly increases as $p_{w}$ is decreased. Therefore, by decreasing $p_{x}$ to $p_{x}^{\prime}$ it must be possible to find a price vector $p^{\prime}$ and an assignment such that 
either $p_{x}^{\prime}>r_{x}$ and $\# N_{x}=q_{x}$ or $p_{x}^{\prime}=r_{x}$ and $\# N_{x} \leq q_{x}$. Furthermore, the demand for the other item $y \in a b$, for which $y \neq x$, has weakly decreased. Therefore, $\# N_{y} \leq q_{y}, p_{y}^{\prime} \geq r_{y}$. Moreover, $\mu(i) \in D_{i}\left(p^{\prime}\right)$ for all $i \in N$ as there would otherwise exist excess demand for item $x$, which could be eliminated by raising its price, as there was no excess demand at $p$. If $\# N_{y}<q_{y}$ and $p_{y}^{\prime}>r_{y}$, then the price of item $y$ can be decreased in the same manner. By repeating this process, it must be possible to find some $p^{\prime} \leq p$, where an assignment can be constructed, such that $\mu(i) \in D_{i}\left(p^{\prime}\right)$ for all $i \in N$ and $\# N_{x}=q_{x}$ for any $x \in a b$ for which $p_{x}^{\prime}>r_{x}$ and $\# N_{x} \leq q_{x}$ for any $x \in a b$ for which $p_{x}^{\prime}=r_{x}$. This implies however that $p^{\prime} \in \mathcal{P}$, contradicting the minimality of $p^{\text {min }}$. There therefore exists $i \in N$ such that $\mu(i) \notin D_{i}(p)$ and by Proposition 5 it follows that $K_{N}(x, p)>q_{x}$ for some package $x \in \mathcal{I}$ and since $K_{N}(0, p) \leq q_{0}$ for all $p$ it must be that $O_{*}(p) \neq 0$.

For Lemma 8 let $x \neq y$ for $x, y \in a b$.

Lemma 8 Iffor any two price vectors $p$ and $p^{\prime}$ where $p_{x}^{\prime}>p_{x}, p_{y}^{\prime}=p_{y}$, and $y \subseteq w$ for all $w \in D_{i}(p)$ and some $i \in N$, then $y \subseteq w$ for all $w \in D_{i}\left(p^{\prime}\right)$

Proof By symmetry it is enough to consider when $x=a$ and $y=b$. If $b \in D_{i}(p)$ for any $i \in N$, then $\left(b, p_{b}^{\prime}\right) \succ_{i}\left(k, p_{k}^{\prime}\right)$ for all $k \in \mathcal{I} \backslash b$ by price monotonicity. If $a b=D_{i}(p)$, then $f_{2}\left(p_{a}\right)=p_{b}^{2}>p_{b}$ by price monotonicity. If, to derive a contradiction, $a \in D_{i}\left(p^{\prime}\right)$, then $f_{2}\left(p_{a}^{\prime}\right)=p_{b}^{\prime 2} \leq p_{b}^{\prime}=p_{b}$ and $m_{2}=\frac{p_{b}^{\prime 2}-p_{b}^{2}}{p_{a}^{\prime}-p_{a}}<0$. Let $p^{\prime \prime}$ be a price vector where $p_{a}^{\prime \prime}=p_{a}$ and $p_{b}^{\prime \prime}=p_{b}+\gamma$ for some $\gamma>0$ sufficiently small such that $D_{i}\left(p^{\prime \prime}\right)=a b$ as well. As $m_{2}<0$ there exists a price vector $k$, for which $k_{a}<p_{a}^{\prime}$ and $k_{b}=p_{b}^{\prime \prime}$, where $f_{2}\left(k_{a}\right)=k_{b}^{2}<k_{b}$ and hence $\left(a, k_{a}\right) \succ_{i}\left(a b, k_{a b}\right)$. Moreover, as $a \in D_{i}\left(p^{\prime}\right)$ and $k_{a}<p_{a}^{\prime}$ and $k_{b}>p_{b}^{\prime}$ we must by price monotonicity have $\left(a, k_{a}\right) \succ_{i}\left(x, p_{x}\right)$ for $x \in\{b, 0\}$ as well. Hence, $D_{i}(k)=a$, which contradicts the gross substitutes condition since $b \notin w$ for any $w \in D_{i}(l)$.

Now we will show that $a b=D_{i}(p)$ implies that $\left(b, p_{b}^{\prime}\right) \succ_{i}(0,0)$. Assume $(0,0) \succsim_{i}$ $\left(b, p_{b}^{\prime}\right)$, which by price monotonicity implies that $p_{b}^{\prime}=p_{b} \geq v_{b}$. For some price vector $k$ such that $k_{b}=p_{b}+\gamma$ and $k_{a}=p_{a}$ for some $\gamma>0$ sufficiently small we must have $D_{i}(k)=a b$ as well. Let $k^{\prime}$ be a price vector where $k_{b}^{\prime}=k_{b}$ and $k_{a}^{\prime}>k_{a}$ such that $k_{b}^{\prime}+k_{a}^{\prime}>v_{a b}$. From the previous arguments we know that $a \notin D_{i}\left(k^{\prime}\right)$. Therefore, $0=D_{i}\left(k^{\prime}\right)$. This however, violates the gross substitutes condition since $b \notin w$ for any $w \in D_{i}\left(k^{\prime}\right)$. Hence, $\left(b, k_{b}\right) \succ_{i}(0,0)$, which concludes the proof.

Lemma $9 p^{T} \leq p^{\text {min }}$

Proof To derive a contradiction assume that $p^{t} \leq p^{\text {min }}$ for some $t<T$ but $p_{x}^{t+1}>p_{x}^{\text {min }}$ for some $x \in a b$. Denote the unique minimal set in excess demand at time $t$ by $O_{*}\left(p^{t}\right)$. We know that there must exist some $t$ and $e \in[0, \varepsilon(t))$ such that $p^{\prime}(e)=p^{t}+e \delta\left(p^{t}\right) \leq p^{\text {min }}$. As $e<\varepsilon(t)$, it follows that $O_{*}\left(p^{t}\right)=O_{*}\left(p^{\prime}(e)\right) \neq 0$. Let $c(p)=\left\{x \in a b \mid p_{x}=p_{x}^{\text {min }}\right\}$ for any $p$. Moreover, let $c_{1}=O_{*}\left(p^{\prime}(e)\right) \cap c\left(p^{\prime}(e)\right)$ and $c_{2}=O_{*}\left(p^{\prime}(e)\right) \backslash c_{1}$. We start by noting that if $g\left(x, p^{\prime}(e)\right)>0$ for $x \in a b$, then $K_{N}(x, p)=\# \mathcal{D}_{x}\left(p^{\prime}(e)\right)>q_{x}$. There are two cases to consider:

Case $1 c_{1} \neq \varnothing$. If $g\left(c_{1}, p^{\prime}(e)\right)>0$, then either $c_{1}=a b$, in which case $p^{\text {min }} \notin \mathcal{P}$, or $c_{1} \in a b$, which implies that $K_{N}\left(c_{1}, p^{\prime}(e)\right)=\# \mathcal{D}_{c_{1}}\left(p^{\prime}(e)\right)>q_{c_{1}}$. As $c_{1} \subseteq w$ for all $w \in D_{i}\left(p^{\prime}(e)\right)$ for all $i \in \mathcal{D}_{c_{1}}\left(p^{\prime}(e)\right)$, it follows by Lemma 8 that $c_{1} \subseteq w$ for all $w \in D_{i}\left(p^{\text {min }}\right)$ for any such bidder $i$ as well. Therefore, $K_{N}\left(c_{1}, p^{\text {min }}\right) \geq K_{N}\left(c_{1}, p^{\prime}(e)\right)$ and hence $g\left(c_{1}, p^{\text {min }}\right)>0$, which contradicts that $p^{\text {min }} \in \mathcal{P}$.

Now assume that $g\left(c_{1}, p^{\prime}(e)\right) \leq 0$, which implies that $c_{1} \in a b$ and $O_{*}\left(p^{\prime}(e)\right)=a b$. To simplify let $c_{1}=a$ and $c_{2}=b$. By symmetry, the following arguments can be used when 
$a$ and $b$ are interchanged. It will now be shown that $g\left(a, p^{\text {min }}\right)>0$. To see this we start by noting that as $a, b \in D_{i}\left(p^{\prime}(e)\right)$ for all $i \in \mathcal{D}_{a, b}\left(p^{\prime}(e)\right)$, it follows that $K_{i}\left(a b, p^{\prime}(e)\right)=1$ for any such bidder $i \in N$. Therefore, it follows that $g\left(a b, p^{\prime}(e)\right)=\# \mathcal{D}_{a, b}\left(p^{\prime}(e)\right)+g\left(a, p^{\prime}(e)\right)+$ $g\left(b, p^{\prime}(e)\right)$ and we know that $\# \mathcal{D}_{a, b}\left(p^{\prime}(e)\right) \geq 1$ since $O_{*}\left(p^{\prime}(e)\right)=a b$ and $g\left(a, p^{\prime}(e)\right) \leq 0$.

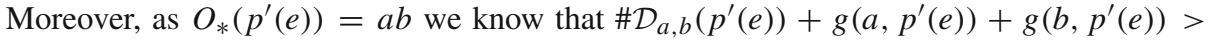
$g\left(b, p^{\prime}(e)\right)$ or $\# \mathcal{D}_{a, b}\left(p^{\prime}(e)\right)+g\left(a, p^{\prime}(e)\right)>0$. By gross substitutes and price monotonicity it must be that $K_{i}\left(a, p^{\text {min }}\right) \geq K_{i}\left(a,\left(p^{\prime}(e)\right)\right)$ for all $i \in N$. In particular, since $a, b \in$ $D_{i}\left(p^{\prime}(e)\right)$ for all $i \in \mathcal{D}_{a, b}\left(p^{\prime}(e)\right)$, it follows that $K_{i}\left(a, p^{\prime}(e)\right)=0$ and by gross substitutes and price monotonicity that $K_{i}\left(a, p^{\text {min }}\right)=1$ for any such bidder $i \in \mathcal{D}_{a, b}\left(p^{\prime}(e)\right)$. As $\# \mathcal{D}_{a, b}\left(p^{\prime}(e)\right)+g\left(a, p^{\prime}(e)\right)>0$, it must be that $g\left(a, p^{\text {min }}\right) \geq \# \mathcal{D}_{a, b}\left(p^{\prime}(e)\right)+g\left(a, p^{\prime}(e)\right)>0$, which is a contradiction.

Case 2: $c_{1}=\emptyset$ and $c\left(p^{\prime}(e)\right) \neq \emptyset$. As $c_{1}=\emptyset$ and $c\left(p^{\prime}(e)\right) \neq \emptyset$ it must be that $e, \delta\left(p^{t}\right)$ and $\varepsilon(t)$ are generated in step 3 of Process 1. Furthermore, $c_{2}=O_{*}\left(p^{\prime}(e)\right) \neq \emptyset$ and $O_{*}\left(p^{\prime}(e)\right) \neq$ $a b$ because if $O_{*}\left(p^{\prime}(e)\right)=a b$, then $c_{1} \neq \emptyset$. For simplicity we can let $c_{2}=O_{*}\left(p^{\prime}(e)\right)=a$ but symmetric arguments apply if $c_{2}=b$. Let $p^{\prime \prime}$ be defined as $p_{b}^{\prime \prime}=p_{b}^{\text {min }}=p_{b}^{\prime}(e)$ and $p_{a}^{\prime \prime}=p_{a}^{\prime}(e)+\gamma$ for some $\gamma>0$ sufficiently small such that $p_{a}^{\prime \prime}<p_{a}^{\text {min }}$. As $e$ was generated in step 3 and $O_{*}\left(p^{t}\right)=a=O_{*}\left(p^{\prime}(e)\right)$, we know that $\delta_{0}=0, \delta_{a}\left(p^{t}\right)=1$, and $\delta_{b}\left(p^{t}\right)=l_{b}(t)$, where $l_{b}(t)=\min \left\{\delta_{b}\left(p^{t}\right) \in \mathbb{R}_{+} \mid \delta_{0}\left(p^{t}\right)=0, \delta_{a}\left(p^{t}\right)=1\right.$, and $\left.\varepsilon(t)>0\right\}$. More importantly, as $\varepsilon(t)=0$ in step 2 of Process $1, O_{*}\left(p^{\prime}(e)\right) \neq O_{*}\left(p^{\prime \prime}\right)$.

Note that as $p_{b}^{\prime \prime}=p_{b}^{\text {min }}$ and $p_{a}^{\prime \prime}<p_{a}^{\text {min }}$, we know by Lemma 7 that $O_{*}\left(p^{\prime \prime}\right) \neq 0$. If $O_{*}\left(p^{\prime \prime}\right)=b$, then $p^{\text {min }} \notin \mathcal{P}$ as $g\left(b, p^{\min }\right)>0$ by the gross substitutes condition. Thus, $O_{*}\left(p^{\prime \prime}\right)=a b$, which implies that $g\left(a b, p^{\prime \prime}\right)>g\left(a, p^{\prime \prime}\right)$ or \#- $\mathcal{D}_{a, b}\left(p^{\prime \prime}\right)+g\left(a, p^{\prime \prime}\right)+$ $g\left(b, p^{\prime \prime}\right)>g\left(a, p^{\prime \prime}\right)$ and hence $\# \mathcal{D}_{a, b}\left(p^{\prime \prime}\right)+g\left(b, p^{\prime \prime}\right)>0$. Since $a, b \in D_{i}\left(p^{\prime \prime}\right)$ for all $i \in \mathcal{D}_{a, b}\left(p^{\prime \prime}\right)$ we know by price monotonicity that $a \notin D_{i}\left(p^{\text {min }}\right)$ and by Lemma 8 that $b \in D_{i}\left(p^{\text {min }}\right)$ for all $i \in \mathcal{D}_{a, b}\left(p^{\prime \prime}\right)$ as well. Furthermore, $K_{i}\left(b, p^{\text {min }}\right) \geq K_{i}\left(b, p^{\prime \prime}\right)$ for any $i \in N \backslash \mathcal{D}_{a, b}\left(p^{\prime \prime}\right)$. Therefore, $g\left(b, p^{\text {min }}\right)>0$, and/or $g\left(a b, p^{\text {min }}\right)>0$, which contradicts that $p^{\text {min }} \in \mathcal{P}$.

Theorem 1 Process 1 always terminates at $p^{T}=p^{\text {min }}$.

Proof Lemmas 7 and 9 together imply Theorem 1.

\section{References}

Andersson, T., Andersson, C., \& Talman, A. (2013). Sets in excess demand in simple ascending auctions with unit-demand bidders. Annals of Operations Research, 211(1), 27-36.

Andersson, T., \& Erlanson, A. (2013). Multi-item Vickrey-English-Dutch auctions. Games and Economic Behavior, 81, 116-129.

Ausubel, L. (2004). An efficient ascending-bid auction for multiple objects. American Economic Review, 94, $1452-1475$.

Ausubel, L. (2006). An efficient dynamic auction for heterogeneous commodities. American Economic Review, 96(5), 1-28.

Ausubel, L., \& Milgrom, P. (2002). Ascending auctions with package bidding. Advances in Theoretical Economics, 1(1).

Binmore, K., \& Klemperer, P. (2002). The biggest auction ever: The sale of the British $3 \mathrm{~g}$ telecom licences. The Economic Journal, 112(478), C74-C96.

de Vries, S., Schummer, J., \& Vohra, R. (2007). On ascending vickrey auctions for heterogeneous objects. Journal of Economic Theory, 132(1), 95-118.

Demange, G., \& Gale, D. (1985). The strategy structure of two-sided matching markets. Econometrica, 53(4), 873-888.

Demange, G., Gale, D., \& Sotomayor, M. (1986). Multi-item auctions. Journal of Political Economy, 94(4), 863-872. 
Erlanson, A. (2014). The duo-item bisection auction. Computational Economics, 43(1), 15-31.

Grigorieva, E., Herings, P. J., Müller, R., \& Vermeulen, D. (2007). The private value single item bisection auction. Economic Theory, 30(1), 107-118.

Gul, F., \& Stacchetti, E. (1999). Walrasian equilibrium with gross substitutes. Journal of Economic Theory, 87(1), 95-124.

Gul, F., \& Stacchetti, E. (2000). The English auction with differentiated commodities. Journal of Economic Theory, 92(1), 66-95.

Hatfield, J., \& Milgrom, P. (2005). Matching with contracts. American Economic Review, 94(4), 913-935.

Kelso, A., \& Crawford, V. (1982). Job matching, coalition formation, and gross substitutes. Econometrica, 50(6), 1483-1504.

Klemperer, P. (2010). The product-mix auction: A new auction design for differentiated goods. Journal of the European Economic Association, 8(2-3), 526-536.

Maskin, E., \& Riley, J. (1984). Optimal auctions with risk averse buyers. Econometrica, 52(6), 1473-1518.

Matthews, S. (1987). Comparing auctions for risk averse buyers: A buyer's point of view. Econometrica, 55(3), 633-646.

Mezzetti, C. (2011). Sequential auctions with informational externalities and aversion to price risk: Decreasing and increasing price sequences. The Economic Journal, 121(555), 990-1016.

Milgrom, P. (2000). Putting auction theory to work: The simultaneous ascending auction. Journal of Political Economy, 108(2), 245-272.

Mishra, D., \& Parkes, D. (2007). Ascending price vickrey auctions for general valuations. Journal of Economic Theory, 132(1), 335-366.

Mishra, D., \& Talman, D. (2010). Characterization of the walrasian equilibria of the assignment model. Journal of Mathematical Economics, 46(1), 6-20.

Morimoto, S., \& Serizawa, S. (2015). Strategy-proofness and efficiency with non-quasi-linear preferences: A characterization of minimum price walrasian rule. Theoretical Economics, 10(2), 445-487.

Sankaran, J. (1994). On a dynamic auction mechanism for a bilateral assignment problem. Mathematical Social Sciences, 28(2), 143-150.

Shapley, L., \& Shubik, M. (1972). The assignment game i: The core. International Journal of Game Theory, 1(1), 111-130.

Sun, N., \& Yang, Z. (2006). Equilibria and indivisibilities: Gross substitutes and complements. Econometrica, 74(5), 1385-1402.

Sun, N., \& Yang, Z. (2009). A double-track adjustment process for discrete markets with substitutes and complements. Econometrica, 77(3), 933-952.

Sun, N., \& Yang, Z. (2014). An efficient and incentive compatible dynamic auction for multiple complements. Journal of Political Economy, 122(2), 422-466.

Publisher's Note Springer Nature remains neutral with regard to jurisdictional claims in published maps and institutional affiliations. 